BULLETIN (New Series) OF THE

AMERICAN MATHEMATICAL SOCIETY

Volume 38, Number 2, Pages 171-216

S 0273-0979(01)00894-1

Article electronically published on January 9, 2001

\title{
NONCOMMUTATIVE CURVES AND NONCOMMUTATIVE SURFACES
}

\author{
J. T. STAFFORD AND M. VAN DEN BERGH
}

\begin{abstract}
In this survey article we describe some geometric results in the theory of noncommutative rings and, more generally, in the theory of abelian categories.

Roughly speaking and by analogy with the commutative situation, the category of graded modules modulo torsion over a noncommutative graded ring of quadratic, respectively cubic, growth should be thought of as the noncommutative analogue of a projective curve, respectively surface. This intuition has led to a remarkable number of nontrivial insights and results in noncommutative algebra. Indeed, the problem of classifying noncommutative curves (and noncommutative graded rings of quadratic growth) can be regarded as settled. Despite the fact that no classification of noncommutative surfaces is in sight, a rich body of nontrivial examples and techniques, including blowing up and down, has been developed.
\end{abstract}

\section{Contents}

1. Introduction 172

2. Geometric constructions 177

3. Twisted homogeneous coordinate rings 180

4. Algebras with linear growth 182

5. Noncommutative projective curves I: Domains of quadratic growth

6. Noncommutative projective curves II: Prime rings 186

7. Noncommutative smooth proper curves 189

8. Artin-Schelter regular algebras 194

9. Commutative surfaces 198

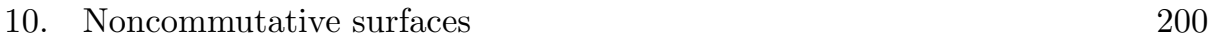

11. Noncommutative projective planes and quadrics 202

12. $\mathbb{P}^{1}$-Bundles $\quad 206$

13. Noncommutative blowing up 208

\begin{tabular}{l|l} 
Index & 213 \\
\hline
\end{tabular}

\begin{tabular}{ll} 
References & 213 \\
\hline
\end{tabular}

Received by the editors October 18, 1999, and in revised form May 20, 2000.

2000 Mathematics Subject Classification. Primary 14A22, 14F05, 16D90, 16P40, 16S80, 16W50, 18E15.

Key words and phrases. Noetherian graded rings, noncommutative projective geometry, deformations, twisted homogeneous coordinate rings.

The first author was supported in part by an NSF grant.

The second author is a senior researcher at the FWO and was partially supported by the Clay Research Institute during the preparation of this article. 


\section{INTRODUCTION}

It has always been clear that noncommutative rings are much more complicated than commutative ones. In order to quantify this feeling Mike Artin and Bill Schelter started a project about 15 years ago which aimed at completely classifying the objects in at least some non-trivial classes of noncommutative rings, the major class being the rings now called Artin-Schelter regular rings of dimension three (see Section 8). Algebraic geometry does not generalize naïvely to noncommutative algebra, yet, remarkably, one can still carry across much of the intuition and technique. One can think of these techniques as constituting a kind of noncommutative algebraic geometry. The present article constitutes an introduction to this field.

This theory stays close in spirit to the classical theory of algebraic geometry as described in textbooks such as [26], 65. There are many other approaches, some of which will be mentioned at the end of the introduction. Our guiding theme will be to classify or at least understand rings of small dimension, just as one natural approach to algebraic geometry would be to emphasize curves and surfaces. As in the classical case, this will also force us to use more abstract notions and these will be introduced as needed. Curiously, it seems that the most substantial applications of this principle occur with projective rather than affine geometry. This is perhaps due to the fact that, as localization is rarely available in the noncommutative universe, most of the affine techniques do not work, whereas the more global and categorical approaches to projective geometry can be generalized. Thus we will restrict our attention to graded rings.

To begin with think about the commutative case. We assume throughout that $k$ is an algebraically closed field and that all rings are $k$-algebras. Given a finitely generated, commutative graded $k$-algebra $C=k\left[x_{1}, \ldots, x_{n}\right] / I$ that is generated in degree one, the zeroes of $I$ yield an associated projective scheme $X=\operatorname{Proj}(C) \subseteq$ $\mathbb{P}^{n-1}$. Conversely, given $X$ one can recover $C$ up to a finite dimensional vector space by defining $C_{n}$ to be the functions on $X$ with a pole of order at most $n$ at infinity. Crucially, the modules over $X$ and $C$ are closely related; the category $\operatorname{coh}(X)$ of coherent sheaves on $X$ is equivalent to the quotient category $\operatorname{qgr}(C)$ of finitely generated, graded $C$-modules modulo those of finite length (see Theorem 2.1 .2 for the precise statement). In what follows we will regard $\operatorname{coh}(X)$ rather than $X$ as the scheme, since this is what generalizes. It can also be justified by Rosenberg's reconstruction theorem 61 .

Now consider a finitely generated, noncommutative graded $k$-algebra $R$. Obviously $R$ can be written as a factor ring $T / I$ of a free algebra $T$, but this is not very useful since such a presentation gives virtually no insight into the structure of $R$ and certainly does not seem to lead to any geometric insight.

One therefore needs a more geometric way of studying such algebras. Fortunately the categorical aspects of the commutative theory do generalize, and one of the major themes of this article will be to work not just with $R$ but also with $\operatorname{qgr}(R)$, where these more geometric techniques apply. Crucially, one can go back and forth:

- (a) One can determine when a category $\mathcal{C}$ is isomorphic to $\operatorname{qgr}(R)$ for a noetherian ring $R$ (combine Theorems 2.1.5 and 1.1.1).

(b) For a large class of algebras one can recover $R$, at least up to a finite dimensional vector space, from data in $\operatorname{qgr}(R)$ (see Theorem [2.1.6).

(c) Simple conditions on $R$ are known for $\operatorname{qgr}(R)$ to satisfy geometric conditions like Serre duality (see Section 7.4). 
The most important special case of part (a) and the fundamental construction in the subject involves a noncommutative analogue of the homogeneous coordinate ring of a projective scheme $X$. This is called the twisted homogeneous coordinate ring of $X$ and is defined as follows: Given an automorphism $\sigma$ of $X$ and an invertible sheaf $\mathcal{L}$, the associated twisted homogeneous coordinate ring is defined to be the ring

$$
B(X, \mathcal{L}, \sigma)=\bigoplus_{i \geq 0} \mathrm{H}^{0}\left(X, \mathcal{L} \otimes \sigma^{*} \mathcal{L} \otimes \cdots \otimes\left(\sigma^{n-1}\right)^{*} \mathcal{L}\right),
$$

with a natural multiplication (see Section 3 ). Here, $\tau^{*} \mathcal{L}$ denotes the pull-back of $\mathcal{L}$ along the automorphism $\tau$. Despite the fact that $B(X, \mathcal{L}, \sigma)$ is far from commutative, one still has $\operatorname{qgr}(B(X, \mathcal{L}, \sigma)) \simeq \operatorname{coh}(X)$ when $\mathcal{L}$ satisfies the appropriate form of ampleness. For example, the two most basic noncommutative graded rings of quadratic growth are the quantum (affine) planes

$$
U_{\infty}=k\{x, y\} /\left(x y-y x-x^{2}\right) \quad \text { and } \quad U_{q}=k\{x, y\} /(x y-q y x), \quad \text { for } q \in k^{*} .
$$

These can both be written as $B\left(\mathbb{P}^{1}, \mathcal{O}_{\mathbb{P}^{1}}(1), \sigma\right)$, where $\sigma$ is defined, respectively, by $\sigma(u)=u+1$ and $\sigma(u)=q u$ on $k\left(\mathbb{P}^{1}\right)=k(u)$. For both rings $U$ one therefore has $\operatorname{qgr}(U) \cong \operatorname{coh}\left(\mathbb{P}^{1}\right)$.

Twisted homogeneous coordinate rings seem to be pervasive in the geometrical approach to noncommutative rings being promoted in this survey. They are fundamental in the classification of noncommutative curves. Perhaps more surprisingly, many graded rings $R$ come equipped with a canonical map to a twisted homogeneous coordinate ring $B$ (see Section 8 ) and that coordinate ring then forms the starting point in the study of the original ring $R$ (see Sections 8 and 13).

Let us now consider noncommutative curves. The first question is to find the appropriate definition of such an object. There are two possible answers, both of which play a rôle in this subject. The first is algebraic and asserts that, just as in the commutative case, a curve is the abelian category $\operatorname{qgr}(R)$ associated to a graded ring $R=\bigoplus_{i \geq 0} R_{i}$ with quadratic growth; thus $\operatorname{dim}_{k}\left(R_{i}\right)$ grows linearly. The rings $U_{\infty}$ and $U_{q}$ would be typical examples. The second answer is geometric and asserts that a smooth noncommutative curve should be an abelian category $\mathcal{C}$ with the basic properties of $\operatorname{coh}(E)$ for a smooth projective curve $E$. These analogies work rather well:

- let $R$ be a graded domain of quadratic growth, and assume that $R$ is generated by $R_{1}$ as an algebra. Then, up to a finite dimensional vector space, $R \cong$ $B(E, \mathcal{L}, \sigma)$, for some curve $E$. Moreover, the corresponding categories $\operatorname{coh}(E)$ and $\operatorname{qgr}(R)$ are equivalent (see Theorem 5.2 and Corollary 5.3).

This result allows one to give a detailed description of the structure of $R$; for example, if $\sigma$ has finite order, then $R$ will be finitely generated as a module over its centre, while if $\sigma$ has infinite order, then $R$ has a faithful irreducible representation (see Corollary 5.4). When $R$ is not generated in degree one, the situation is somewhat more subtle although, under mild assumptions, $R$ can still be classified in terms of a twisted coordinate ring $B(E, \mathcal{L}, \sigma)$, and the categories $\operatorname{coh}(E)$ and $\operatorname{qgr}(R)$ will still be equivalent. See Sections 5 and 6 for the details.

On the other hand, if one considers a category $\mathcal{C}$ with the basic properties of $\operatorname{coh}(E)$, then: 
- such an abelian category $\mathcal{C}$ is equivalent to $\operatorname{qgr}(R)$ for the appropriate prime ring $R$ of quadratic growth (see Section 7 ).

These results give a precise formulation of the intuitive picture of noncommutative curves, and we can regard this topic as being essentially settled.

The second half of the survey considers noncommutative surfaces. Despite the fact that no form of classification is in sight, a rich body of nontrivial examples and techniques has been developed that should be useful, not only for studying these surfaces but possibly also for more general algebraic questions.

For the purposes of this introduction, define a noncommutative irreducible surface to be $\operatorname{qgr}(R)$ for a graded domain $R$ of cubic growth, as would be the case for commutative surfaces (see Section 10 for a more detailed discussion of the appropriate definition). In the commutative case, which is briefly reviewed in Section 9. the simplest example is the projective plane $\mathbb{P}^{2}=\operatorname{Proj}(k[x, y, z])$. As we show (see Section [1) there are strong arguments for saying that:

- the noncommutative projective planes are $\operatorname{qgr}(A)$, for the so-called ArtinSchelter regular algebras $A$ of dimension 3 with Hilbert series $(1-t)^{-3}$ (see Section (8). These rings have been completely classified.

Even in dimension three, these Artin-Schelter algebras are more diverse and have a more complicated structure than might be expected. The obvious examples are the analogues of the quantum affine planes mentioned earlier, the best known one being the $k$-algebra with generators $\left\{x_{i}: 1 \leq i \leq 3\right\}$ which " $q$-commute" in the sense that $x_{i} x_{j}=q_{i j} x_{j} x_{i}$, for $i<j$ and $q_{i j} \in k^{*}$. However the generic example is the Sklyanin algebra $\mathrm{Skl}_{3}$ (see Example 8.3 et seq) and this is much more subtle. In particular, it has a factor ring isomorphic to $B(E, \mathcal{L}, \sigma)$, where $\sigma$ is an automorphism of a smooth elliptic curve $E$. Geometrically, this can be interpreted as saying that the noncommutative projective plane $\mathrm{qgr}\left(\mathrm{Skl}_{3}\right)$ has an embedded commutative elliptic curve $\operatorname{coh}(E)$. The algebra $\mathrm{Skl}_{3}$ is actually determined by the data $(E, \mathcal{L}, \sigma)$ (the analogous statement holds for all Artin-Schelter algebras of dimension three) and so, even here, the noncommutative space is strongly controlled by a commutative variety via a twisted coordinate ring.

The standard classification of smooth projective surfaces has three parts: first one classifies surfaces up to birational equivalence (this just means that one classifies the fields of transcendence degree two over $k$ ); secondly one has Zariski's theorem that asserts that one can pass from any smooth projective surface to a birationally equivalent one by successive blowing up and down; thirdly one needs to know the minimal models within each such equivalence class. The minimal models for rational surfaces are $\mathbb{P}^{2}, \mathbb{P}^{1} \times \mathbb{P}^{1}$ and $\mathbb{P}^{1}$-bundles over $\mathbb{P}^{1}$. As mentioned in the last paragraph, we know the noncommutative analogues for $\mathbb{P}^{2}$ and

- the noncommutative analogues of $\mathbb{P}^{1} \times \mathbb{P}^{1}$ and $\mathbb{P}^{1}$-bundles over $\mathbb{P}^{1}$ can also be determined (see Sections 11 and 12);

Moreover:

- There even exists a noncommutative version of blowing up and down (see Section 13).

This is considerably more subtle than the commutative analogue since we need to blow up at one-sided rather than at two-sided ideals and the arguments therefore have to be categorical rather than ring-theoretic. Nevertheless, the end result is surprisingly similar to the commutative version. We illustrate this by showing that: 
- the standard constructions of the Cremona transformation, cubic surfaces and quadric surfaces via blowing up/down generalize completely to noncommutative surfaces.

This can be seen by comparing the noncommutative versions in (13.3-13.5) with their commutative analogues, as outlined in (9.2 9.4).

As was mentioned earlier, there is no complete classification for noncommutative surfaces and, in particular, there is as yet no analogue of the classification of fields of transcendence degree two nor of Zariski's theorem. This will hopefully form a fruitful area of research for the reader.

There are many different approaches to classical geometry and - at least as yetthese become more diverse when one considers their noncommutative (or quantum or deformed) analogues. As we have argued above, the theory of projective curves and surfaces generalizes surprisingly effectively (see [33] for an application to the study of noncommutative instantons). One also has the seminal work by A. Connes on what is essentially noncommutative differential geometry. Similarly, much of the theory of quantum groups can be thought of as a noncommutative geometry, more precisely as a noncommutative analogue of the theory of algebraic groups (see, for example, [46]). Just as differential geometry and the theory of algebraic groups tend to be rather different from classical algebraic geometry, so their noncommutative counterparts are distinct.

Other variants of noncommutative geometry can be found in [12, [16], [39], [41], [47, 60], [91] (this is by no means an exhaustive list), and we will end the introduction by briefly discussing a few of these approaches to noncommutative algebraic geometry.

Equating noncommutative schemes with abelian categories, as we have done, has drawbacks: most notably there is no good general notion of vector bundle. In the recent preprint by Kontsevich and Rosenberg [39] this defect is elegantly repaired by introducing a suitable notion of covering (see also [92]). Unfortunately the covering formalism creates other difficulties, and it is not clear whether a noncommutative graded ring should give rise to a noncommutative scheme in their sense.

Many approaches to noncommutative algebraic geometry may eventually be incorporated into a theory of deformations of orders over varieties. However, this first requires a thorough understanding of the geometry of such orders or, more generally, of rings satisfying a polynomial identity. For the latter the reader is referred to 91]. Of course, the fundamental property of rings satisfying a polynomial identity is that their irreducible representations are finite dimensional, and for a general theory of the moduli spaces of finite dimensional representations of more general rings the reader is referred to 41, 442. Closely related is Kapranov's notion of a noncommutative "thickening" of a smooth commutative variety [32. Many of the questions studied in these papers are related to Kontsevich's suggestion that many moduli problems could be reformulated as a study of the finite dimensional representations of infinite dimensional algebras.

Arguably [16] one should generalize further and work directly with triangulated categories. However, due to a certain flabbiness in their definition this can create serious technical problems that are quite unrelated to the underlying geometric intuition. It is now clear that most of these problems can be solved by considering DG-categories [35] or, more generally, $\mathrm{A}_{\infty}$ categories [36], so it seems worthwhile to pursue this viewpoint further. 
Acknowledgement. We would like to thank Dennis Keeler and Haynes Miller for many suggestions that improved the exposition of this article.

1.1. Notation. Here, we collect some standard notation that will be in force throughout the paper.

Fix once and for all a base field $k$, which will be algebraically closed unless we say otherwise. All objects and morphisms will be assumed to be $k$-linear. Thus all rings will be $k$-algebras, all schemes will be $k$-schemes, all additive categories will be $k$ linear, etc. If $A$ is a ring, then $\operatorname{Mod}(A)$ will denote the category of right $A$-modules. Similarly, if $R=\bigoplus_{i \in \mathbb{Z}} R_{i}$ is a $\mathbb{Z}$-graded ring, then $\operatorname{Gr}(R)$ will be the category of $\mathbb{Z}$-graded right $R$-modules. In this category homomorphisms are of degree zero; thus if $M=\bigoplus_{i \in \mathbb{Z}} M_{i}$ and $N=\bigoplus_{i \in \mathbb{Z}} N_{i} \in \operatorname{Gr}(R)$, and $\theta \in \operatorname{Hom}_{\operatorname{Gr}(R)}(M, N)$, then $\theta\left(M_{i}\right) \subseteq N_{i}$ for all $i$. We let Tors $(R)$ denote the full subcategory of $\operatorname{Gr}(R)$ generated by the right-bounded modules, that is, by modules $M=\bigoplus_{i \in \mathbb{Z}} M_{i}$ for which $M_{i}=0$ for $i \gg 0$. Set $\mathrm{QGr}(R)$ for the quotient category $\operatorname{Gr}(R) / \operatorname{Tors}(R)$ (the formal definition of this can be found, for example, in 22 but, roughly speaking, Q $\operatorname{Gr}(R)$ has the same objects as $\operatorname{Gr}(R)$ but objects in Tors $(R)$ become isomorphic to 0$)$. Given a graded module $M=\bigoplus M_{i}$ over a graded ring $R$, define the shift $M(r)$ of $M$ to be the graded module defined by $M(r)_{i}=M_{i+r}$.

For the most part we will work with $\mathbb{N}$-graded algebras $R=\bigoplus_{i>0} R_{i}$ that are finitely generated, with $R_{0}$ finite dimensional as a $k$-vector space. We call these algebras finitely graded for short. A finitely graded ring $R$ will be called connected graded if $R_{0}=k$. The difference between these two concepts is largely technical; in particular, if $R$ is a finitely graded domain, then $R$ is connected graded. For an $\mathbb{N}$-graded algebra $R$ we write $R_{+}$for the two-sided ideal $\sum_{n>0} R_{n}$.

We should emphasize that, although we are interested in noncommutative analogues of geometric concepts, the unadorned terms "scheme", "variety", etc., will always mean the classical (commutative) objects. Let $X$ be a scheme. Then $\mathrm{Qch}(X)$ will denote the category of quasi-coherent $\mathcal{O}_{X}$-modules. If $X$ is noetherian, then we denote by $\operatorname{coh}(X)$ the category of coherent $\mathcal{O}_{X}$-modules. In all cases of practical interest $\mathrm{Q} \operatorname{ch}(X)$ is a so-called Grothendieck category [83]. This is an abelian category with a generator and exact filtered direct limits. Such a category automatically has products and enough injectives [25 Théorème 1.10.1]. Since abelian categories can be embedded in module categories, many standard terms from module theory extend naturally to abelian categories and, on the whole, their definitions will not be given explicitly.

Standard convention. Throughout the paper, if $\mathrm{XYuvw}(\cdots)$ denotes an abelian category, then we will denote by $\operatorname{xyuvw}(\cdots)$ the full subcategory consisting of noetherian objects (an object is noetherian if it satisfies the ascending chain condition on subobjects).

This convention fixes the meaning of $\bmod (A), \operatorname{gr}(R), \operatorname{tors}(R)$ and $\operatorname{qgr}(R)$. Note that, when $R$ is finitely graded, $\operatorname{tors}(R)$ consists of the finite dimensional graded $R$ modules. As is true of $\operatorname{Mod}(R)$ versus $\bmod (R)$, it is sometimes more convenient (and sometimes less) to work with $\mathrm{QGr}(R)$ rather than $\operatorname{qgr}(R)$ or with $\mathrm{Qch}(X)$ rather than $\operatorname{coh}(X)$. The following theorem says that each member of these pairs determines the other, and so we are free to work in whichever is most convenient.

This also works more abstractly. Recall that an abelian category is noetherian if every object is noetherian and the isomorphism classes of objects form a set. Given such a noetherian abelian category $\mathcal{C}$ we may embed $\mathcal{C}$ in its closure $\widetilde{\mathcal{C}}$ under direct 
limits [22]. Then $\widetilde{\mathcal{C}}$ is a Grothendieck category, that is locally noetherian in the sense that it has a family of noetherian generators. Since $\tilde{\mathcal{C}}$ has enough injectives one can, for example, define the derived functors of Hom groups from $\mathcal{C}$ by working in $\widetilde{\mathcal{C}}$.

Theorem 1.1.1. [22, Theorem II.4.1] Let $\mathcal{C}$ be a noetherian abelian category and $\widetilde{\mathcal{C}}$ its closure, as above. Then $\mathcal{C}$ is the set of noetherian objects in $\widetilde{\mathcal{C}}$ and these two categories determine each other up to natural equivalence.

If $\mathcal{C}=\operatorname{coh}(X)$ for a noetherian scheme $X$, then $\widetilde{\mathcal{C}}=\mathrm{Q} \operatorname{ch}(X)$ and if $\mathcal{C}=\operatorname{qgr}(R)$ for a right noetherian graded ring $R$, then $\widetilde{\mathcal{C}}=\mathrm{QGr}(R)$.

Finally, the following ring-theoretic definitions will be used without comment in the survey. A ring $R$ is prime, respectively semiprime, if the product of nonzero ideals is nonzero, respectively the square of every nonzero ideal is nonzero. An ideal $I$ of $R$ is (semi)prime if $R / I$ is (semi)prime. The prime radical $N(R)$ of $R$ is defined to be the intersection of the prime ideals of $R$. A useful fact is that $R$ is semiprime if and only if $N(R)=0$. Given $R$-modules $M \subseteq N$, then $N$ is an essential extension of $M$ if every nonzero submodule of $N$ intersects $M$ nontrivially.

\section{Geometric constructions}

The purpose of this section is to clarify the connection between rings and abelian categories in certain standard cases. The main result (see Theorems 2.1.5 and 2.1.6) determines which categories are equivalent to the category $\mathrm{Q} \operatorname{Gr}(R)$ over a right noetherian ring $R$. This will lead us to the notion of "twisted homogeneous coordinate rings" which will turn out to be a basic tool in the construction and classification of noncommutative curves. A very simple example that may help to illustrate these ideas is given in (3.4).

2.1. Affine and projective schemes. Although our main interest will be projective schemes, let us fix the ideas by first considering the affine case. Let $X$ be an affine scheme and put $A=\Gamma\left(X, \mathcal{O}_{X}\right)$. Then it is classical that $\mathrm{Q} \operatorname{ch}(X)$ is equivalent to $\operatorname{Mod}(A)$. This immediately begs the question of which Grothendieck categories can be written as $\operatorname{Mod}(A)$ for a possibly noncommutative ring $A$. The answer is again classical:

Proposition 2.1.1. 78, Example X.4.2] Let $\mathcal{C}$ be a Grothendieck category with a projective generator $\mathcal{O}$ and assume that $\mathcal{O}$ is small; that is, assume that $\operatorname{Hom}(\mathcal{O},-)$ commutes with direct sums. Then $\mathcal{C} \simeq \operatorname{Mod}(A)$, for $A=\operatorname{End}(\mathcal{O})$.

Now consider the projective case. Let $X$ be a projective scheme with a line bundle $\mathcal{L}$. Then the homogeneous coordinate ring $B=B(X, \mathcal{L})$ associated to $(X, \mathcal{L})$ is defined by the formula $B=\bigoplus_{n \in \mathbb{N}} \Gamma\left(X, \mathcal{L}^{n}\right)$ with obvious multiplication. Similarly if $\mathcal{M}$ is a quasi-coherent sheaf on $X$, then $\Gamma_{h}(\mathcal{M})=\bigoplus_{n \in \mathbb{N}} \Gamma\left(X, \mathcal{M} \otimes \mathcal{L}^{n}\right)$ defines a graded $B$ module. Thus the composition of $\Gamma_{h}$ with the natural projection from $\operatorname{Gr}(B)$ to $\mathrm{QGr}(B)$ yields a functor $\bar{\Gamma}_{h}: \mathrm{Qch}(X) \rightarrow \mathrm{QGr}(B)$. This functor only works well when $\mathcal{L}$ is ample in the sense that, if $\mathcal{F} \in \operatorname{coh}(X)$, then $\mathcal{F} \otimes \mathcal{L}^{\otimes n}$ is generated by global sections for all $n \gg 0$ [26, p.153]. In this case one has the following fundamental result of Serre:

Theorem 2.1.2. 64, Proposition 7.8] (i) Let $\mathcal{L}$ be an ample line bundle on a projective scheme $X$. Then the functor $\bar{\Gamma}_{h}(-)$ defines an equivalence of categories between $\mathrm{Qch}(X)$ and $\mathrm{QGr}(B)$. 
(ii) Conversely, if $R$ is a commutative connected graded $k$-algebra that is generated by $R_{1}$ as a $k$-algebra, then there exists a line bundle $\mathcal{L}$ over $X=\operatorname{Proj}(R)$ such that $R=B(X, \mathcal{L})$, up to a finite dimensional vector space. Once again, $\mathrm{Q} \operatorname{Gr}(R) \simeq$ $\mathrm{Qch}(X)$.

It is therefore natural to think of Grothendieck categories of the form $\mathrm{QGr}(B)$ with $B$ a (possibly noncommutative) graded ring as noncommutative projective schemes. Of course, as always, this sort of intuition is only worthwhile if it leads to something useful, but we hope that this survey shows that it does!

Remark 2.1.3. If $R$ is a connected graded commutative ring that is not generated in degree one, then, typically, $\mathrm{QGr}(R) \neq \mathrm{Q} \operatorname{ch}(\operatorname{Proj}(R))$ and so Theorem 2.1 .2 (ii) fails. For example, if $R$ is a weighted polynomial ring, in the sense that the variables are given degrees other than one, then $\mathrm{QGr}(R)$ clearly has finite homological dimension whereas $\operatorname{Proj}(R)$ is usually singular.

The first obvious question is to determine which Grothendieck categories $\mathcal{C}$ are of the form $\mathrm{QGr}(B)$. As we next explain, this has been solved by Artin and Zhang 9]. See 93 for related results of Verevkin.

In Serre's Theorem, one should regard the equivalence as being controlled by three pieces of geometric data: the category $\mathcal{C}=\mathrm{Q} \operatorname{ch}(X)$, the object $\mathcal{O}_{X}$ and the autoequivalence of $\mathcal{C}$ given by tensoring with $\mathcal{L}$. This has a natural generalization. Let $(\mathcal{C}, \mathcal{O}, s)$ be a triple consisting of a Grothendieck category $\mathcal{C}$, an object $\mathcal{O}$ in $\mathcal{C}$ and an autoequivalence $s$ on $\mathcal{C}$. Associated to this data one can define a homogeneous coordinate ring

$$
\Gamma_{h}(\mathcal{C}, \mathcal{O}, s)=\bigoplus_{n \geq 0} \operatorname{Hom}\left(\mathcal{O}, s^{n} \mathcal{O}\right)
$$

with multiplication $a . b=s^{n}(a) b$ for $a \in \operatorname{Hom}\left(\mathcal{O}, s^{m} \mathcal{O}\right), b \in \operatorname{Hom}\left(\mathcal{O}, s^{n} \mathcal{O}\right)$. To simplify the notation we write $\mathcal{O}(n)$ for $s^{n} \mathcal{O}$.

Definition 2.1.4. Assume that $\mathcal{C}$ is locally noetherian. Let $\mathcal{O} \in \mathrm{Ob}(\mathcal{C})$ be a noetherian object and let $s$ be an autoequivalence of $\mathcal{C}$. Then the pair $(\mathcal{O}, s)$ is called ample if the following conditions hold:

(i) For every noetherian object $A \in \mathcal{C}$ there are positive integers $l_{1}, \ldots, l_{p}$ and an epimorphism from $\bigoplus_{i=1}^{p} \mathcal{O}\left(-l_{i}\right)$ to $A$.

(ii) For every epimorphism between noetherian objects $A \rightarrow B$ the induced map $\operatorname{Hom}(\mathcal{O}(-n), A) \rightarrow \operatorname{Hom}(\mathcal{O}(-n), B)$ is surjective for $n \gg 0$.

When $(\mathcal{C}, \mathcal{O}, s)=\left(\mathrm{Q} \operatorname{ch}(X), \mathcal{O}_{X},-\otimes \mathcal{L}\right)$, this definition of ampleness does reduce to the standard one. Indeed, part (i) of the definition reduces to the standard definition of an ample sheaf, while part (ii) corresponds to part of the homological definition. Moreover Serre's theorem also generalizes, as is shown by the following special case of a result of Artin and Zhang.

Theorem 2.1.5. [9, Theorem 4.5] Assume that $\operatorname{dim}_{k} \operatorname{Hom}(M, N)<\infty$ for all noetherian objects $M, N \in \mathcal{C}$ and let $(\mathcal{O}, s)$ be an ample pair. Then $\mathcal{C} \simeq \operatorname{QGr}(B)$ for $B=\Gamma_{h}(\mathcal{C}, \mathcal{O}, s)$. In addition $B$ is right noetherian.

In this theorem, the functor $\bar{\Gamma}_{h}: \mathcal{C} \rightarrow \mathrm{QGr}(B)$ is provided by an analogue of the functor used in Theorem 2.1.2 For $\mathcal{M} \in \mathrm{Ob}(\mathcal{C})$, define $\bar{\Gamma}_{h}(\mathcal{M})$ to be the image in $\mathrm{QGr}(B)$ of $\Gamma_{h}(\mathcal{M})=\bigoplus \operatorname{Hom}_{n \in \mathbb{N}}\left(\mathcal{O}, s^{n} \mathcal{M}\right) \in \operatorname{Gr}(B)$. 
There is also a converse to this theorem, but this requires an extra hypothesis since $B=\Gamma_{h}(\mathcal{C}, \mathcal{O}, s)$ satisfies the extra condition:

$$
\chi_{1}: \quad \operatorname{dim}_{k} \operatorname{Ext}_{B}^{1}\left(B / B_{+}, M\right)<\infty,
$$

for all finitely generated graded $B$-modules $M$. If we allow this condition, then the natural converse to Theorem 2.1.5 goes through. We let $\pi M$ denote the image in $\mathrm{QGr}(R)$ of $M \in \operatorname{gr}(R)$.

Theorem 2.1.6. 9, Theorem 4.5] Let $B$ be a finitely graded, right noetherian $k$ algebra that satisfies $\chi_{1}$. Set $(\mathcal{C}, \mathcal{O}, s)=(\operatorname{QGr}(B), \pi B,(+1))$, where $(+1)$ is the shift functor defined in 1.1. Then $(\pi B,(+1))$ is ample and $\operatorname{dim}_{k} \operatorname{Hom}_{\mathcal{C}}(M, N)<\infty$ for noetherian objects $M, N \in \mathrm{Ob}(\mathcal{C})$. Moreover, $B=\Gamma_{h}(\mathcal{C}, \mathcal{O}, s)$, up to a finite dimensional vector space.

For a more detailed analysis of this categorical approach we refer the reader to [9], [10].

The intrinsic reason for requiring $\chi_{1}$ in this theorem is as follows: Let $M$ be a finitely generated torsion-free $\mathbb{Z}$-graded module over a finitely graded noetherian ring $B$. Then [9] shows that $\widetilde{M}=\Gamma_{h}(\pi M)$ is an $\mathbb{N}$-graded essential extension of $M$ by a torsion module. Thus, if $\widetilde{M} / M$ is finite dimensional, then $\operatorname{dim}_{k} \operatorname{Ext}_{B}^{1}\left(B / B_{+}, M\right)<\infty$. A significant step in the proof of Theorem 2.1.6] is to prove the converse.

Unfortunately, not all noetherian connected graded rings satisfy $\chi_{1}$. The basic counterexample [76] is provided by

$$
S=k+U y \quad \subset \quad U=k\{x, y\} /\left(x y-y x-x^{2}\right) .
$$

This ring is fundamental to many of the results in this paper and so we should describe its properties in some detail. In the notation of the introduction, $U=U_{\infty}$ is one of the two quantum affine planes and one can construct a similar example using $U_{q}$. As $U$ has basis $\left\{x^{i} y^{j}\right\}$, it follows that $S$ has basis $\left\{1, x^{i} y^{j}: i \geq 0, j \geq 1\right\}$. From the identity $\left(x^{n-1} y\right)(x y)-\left(x^{n} y\right) y=-x^{n+1} y$, it is easy to see that $S$ is generated by $y$ and $x y$. However, notice that, although $U / S$ is infinite dimensional, $U \cdot S_{+}=U y \subset S$. Thus $U / S \subseteq \operatorname{Ext}_{S}^{1}(k, S)$ and so $\chi_{1}$ certainly fails. Finally:

$$
S \text { is (right or left) noetherian if and only if char } k=0 \text {. }
$$

The intrinsic reason why this holds will appear in $₫ 5$, but it is easy to prove directly 76. Theorem 2.3]. Another amusing property of this ring is that the presentation and Hilbert series of $S$ are independent of the characteristic of $k$. Thus (2.4) implies that the noetherian property fails to be preserved under reduction modulo $p$ for any prime $p$ !

Theorem 2.1.6 does not fail too badly for $S$, since the $\operatorname{ring} \Gamma_{h}(\mathrm{QGr}(S), \pi S,(+1))$ obtained from that theorem is simply $U$-this should come as no surprise since the last paragraph shows that $U \subseteq \widetilde{S}_{S}$ and it is easy to see that $\Gamma_{h}(\operatorname{QGr}(U), \pi U,(+1))=$ $U$. However, with more complicated examples one can exhibit nastier behaviour. For example, the polynomial ring $T=S[z]$ satisfies $\Gamma_{h}(\mathrm{QGr}(T), \pi T,(+1))=T$, but Theorem 2.1.6 must still fail, since it fails for the factor ring $S$. What now goes wrong is that the shift functor $s=(+1)$ in $\mathrm{QGr}(T)$ is not ample; specifically, Definition 2.1.4 (ii) fails for the map $T \rightarrow S$ (see [76, Theorem 2.10]).

The condition $\chi_{1}$ and the analogues $\chi_{n}$, obtained by replacing Ext ${ }^{1}$ by $\operatorname{Ext}^{n}$ in its definition, are not well understood, although they do hold for most of the natural 
classes of algebras. They also seem to be fundamental to a deeper understanding of the homological properties of noncommutative graded rings. See 7.4 for further results, questions and references in this direction.

\section{Twisted HOMOGENEOUS COORDINATE RINGS}

One may view (2.1) as a factory for producing graded rings, and the interesting point about Theorem 2.1.5 is that fixing $\mathcal{C}$ but varying $(\mathcal{O}, s)$ will produce many different graded rings $B$ with the same $\mathrm{Q} \operatorname{Gr}(B)$. As far as this survey is concerned, the most interesting examples occur when $\mathcal{C}=\mathrm{Q} \operatorname{ch}(X)$, for a projective scheme $X$. We consider this case in detail in this section. There are now two basic ways of obtaining an autoequivalence of $\mathcal{C}=\mathrm{Q} \operatorname{ch}(X)$ : one can either tensor with a line bundle $\mathcal{L}$ (as happens in the commutative case) or apply $\sigma_{*}$ for $\sigma$ an automorphism of $X$. In the sequel we will simply combine them by considering the composition $s=\sigma_{*}(-\otimes \mathcal{L})$. We will say that $\mathcal{L}$ is $\sigma$-ample if $\left(\mathcal{O}_{X}, s\right)$ is ample in the sense of Definition 2.1.4

Following tradition we will write $B(X, \mathcal{L}, \sigma)$ for $\Gamma_{h}\left(\mathrm{Q} \operatorname{ch}(X), \mathcal{O}_{X}, s\right)$ and call it the twisted homogeneous coordinate ring associated to the pair $(\mathcal{L}, \sigma)$. If $\mathcal{M} \in \mathrm{Q} \operatorname{ch}(X)$, then a trite computation yields

$$
s^{n}(\mathcal{M})=\sigma_{*}^{n}\left(\mathcal{M} \otimes \mathcal{L} \otimes \mathcal{L}^{\sigma} \otimes \cdots \otimes \mathcal{L}^{\sigma^{n-1}}\right)
$$

where $\mathcal{L}^{\tau}$ is shorthand for the pullback $\tau^{*} \mathcal{L}$ along an automorphism $\tau$. From (3.1) one obtains $B(X, \mathcal{L}, \sigma)=\bigoplus_{n} B_{n}$ where

$$
B_{n}=\Gamma\left(X, \mathcal{L}_{n}\right) \quad \text { with } \quad \mathcal{L}_{n}=\mathcal{L} \otimes \mathcal{L}^{\sigma} \otimes \cdots \otimes \mathcal{L}^{\sigma^{n-1}} .
$$

The multiplication on $B=B(Y, \mathcal{L}, \sigma)$ is defined in the obvious way: We have a natural map

$$
\begin{aligned}
B_{n} \otimes_{k} B_{m} & \cong \mathrm{H}^{0}\left(X, \mathcal{L}_{n}\right) \otimes_{k} \mathrm{H}^{0}\left(X, \mathcal{L}_{m}\right) \\
& \cong \mathrm{H}^{0}\left(X, \mathcal{L}_{n}\right) \otimes_{k} \mathrm{H}^{0}\left(X, \mathcal{L}_{m}^{\sigma^{n}}\right) \stackrel{\phi}{\longrightarrow} \mathrm{H}^{0}\left(X, \mathcal{L}_{n+m}\right)=B_{n+m},
\end{aligned}
$$

where the map $\phi$ is obtained by taking global sections of the natural homomorphism $\mathcal{L}_{n} \otimes \mathcal{L}_{m}^{\sigma^{n}} \stackrel{\sim}{\longrightarrow} \mathcal{L}_{n+m}$.

One reason why twisted homogeneous coordinate rings are so important is that they frequently appear as factor rings of noncommutative graded rings; indeed, they satisfy a universal property analogous to passing from a noncommutative ring $A$ to its abelianization $A /[A, A]$. Since this is technical to write out formally, we will refer the reader to $\$ 8$ where this process is illustrated by example, and to 10 for a more thorough treatment.

Of course, this discussion begs the question of precisely which line bundles are $\sigma$-ample. A simple application of the Riemann-Roch theorem shows that,

$$
\text { if } X \text { is a curve, then any ample invertible sheaf is } \sigma \text {-ample, }
$$

and the converse holds for irreducible curves. However, for higher dimensional varieties, the situation is more subtle and is described by the following result, for which we need some notation. Let $X$ be a projective scheme and write $A_{\text {Num }}^{1}(X)$ for the set of Cartier divisors of $X$ modulo numerical equivalence. Let $\sigma$ be an automorphism of $X$ and let $P_{\sigma}$ denote its induced action on $A_{\mathrm{Num}}^{1}(X)$. Since $A_{\text {Num }}^{1}(X)$ is a finitely generated free abelian group [38, Remark 3, p.305], we may 
represent $P_{\sigma}$ by a matrix, and we call $P_{\sigma}$ quasi-unipotent if all the eigenvalues of this matrix are roots of unity.

Theorem 3.1. 34] Let $X$ be a projective scheme and let $\sigma$ be an automorphism of $X$. Then $X$ has a $\sigma$-ample line bundle if and only if $P_{\sigma}$ is quasi-unipotent. If $P_{\sigma}$ is quasi-unipotent, then all ample line bundles are $\sigma$-ample.

One consequence of the proof of this result is that a line bundle $\mathcal{L}$ is $\sigma$-ample if and only if it is $\sigma^{-1}$-ample. This is significant since the opposite $\operatorname{ring} B(X, \mathcal{L}, \sigma)^{\circ} \cong$ $B\left(X, \mathcal{L}, \sigma^{-1}\right)$. Combined with Theorem 2.1.5, this proves:

Theorem 3.2. Let $X$ be a projective scheme with an automorphism $\sigma$ and suppose that $\mathcal{L}$ is a $\sigma$-ample line bundle. Then $B=B(X, \mathcal{L}, \sigma)$ is a left and right noetherian ring for which $\operatorname{qgr}(B) \simeq \operatorname{coh}(X)$.

This result can be interpreted as saying that, although twisted homogeneous coordinate rings are generally very noncommutative, they behave from a geometric standpoint as if they are commutative.

Checking the condition that $P_{\sigma}$ is quasi-unipotent is rather easy in practice. For example, assume that $X$ is a projective variety with a canonical divisor that is either ample or anti-ample. Then $P_{\sigma}$ is quasi-unipotent for every automorphism $\sigma$ [34] Proposition 5.6]. It follows that for varieties like Grassmannians all automorphisms must be quasi-unipotent. Furthermore it looks as if the condition for $P_{\sigma}$ to be quasiunipotent might actually be topological. This is illustrated by the following result due to Mike Artin.

Proposition 3.3. Assume that $X$ is a smooth projective surface over $\mathbb{C}$. Then $P_{\sigma}$ is quasi-unipotent if and only if the action of $\sigma$ is quasi-unipotent on $H^{2}(X, \mathbb{C})$.

Example 3.4. Let us compute the twisted homogeneous coordinate rings in the simplest nontrivial case, when $X$ is the projective line $\mathbb{P}^{1}$ and $\mathcal{L}=\mathcal{O}_{\mathbb{P}^{1}}(1)$. Pick a parameter $u$ for $\mathbb{P}^{1}$, so that the standard affine cover $U=\mathbb{P}^{1} \backslash \infty, V=\mathbb{P}^{1} \backslash 0$ has rings of regular functions $\mathcal{O}(U)=k[u]$ and $\mathcal{O}(V)=k\left[u^{-1}\right]$. Identify $\mathcal{O}_{\mathbb{P}^{1}}(1)$ with the sheaf of functions on $\mathbb{P}^{1}$ that have at most a simple pole at infinity; in other words, it is the sub-sheaf of $k(u)=k\left(\mathbb{P}^{1}\right)$ generated by $\{1, u\}$. Then a simple calculation shows that $\mathrm{H}^{0}\left(X, \mathcal{L}^{\otimes n}\right)$ is spanned by $\left\{1, u, \ldots, u^{n}\right\}$ and that $B(X, \mathcal{L}) \cong k[x, y]$, where $x=1$ and $y=u$, thought of as elements of $B_{1}=\mathrm{H}^{0}(X, \mathcal{L})$.

Now repeat this computation for $B\left(\mathbb{P}^{1}, \mathcal{O}_{\mathbb{P}^{1}}(1), \sigma\right)$ where $\sigma$ is the automorphism defined by $\sigma(u)=u+1$. Here, we use the same symbol to denote both an automorphism of the field of rational functions $K=k(X)$ of a smooth projective curve $X$ and for the automorphism this induces on $X$. Our convention is that $\sigma$ acts on the right on $K$ and on the left on $X$; thus $f^{\sigma}(y)=f(\sigma(y))$, for $f \in K$ and $y \in X$. Then $\mathcal{L}=\mathcal{O}_{\mathbb{P}^{1}}(1)$ satisfies $\mathcal{L}^{\sigma} \cong \mathcal{L}$; indeed under our concrete presentation of $\mathcal{L}$, one even has $\mathcal{L}^{\sigma}=\mathcal{L}$ and hence $\mathcal{L}_{n}=\mathcal{L}^{\otimes n}=\mathcal{O}(n)$. Therefore, $B\left(\mathbb{P}^{1}, \mathcal{L}, \sigma\right)=B\left(\mathbb{P}^{1}, \mathcal{L}\right.$, id $)$, as graded vector spaces. However, the multiplication is twisted: If we again set $x=1$ and $y=u$, as elements of $B_{1}$, then the multiplication map (3.2) sends

$$
y \otimes x=u \otimes 1 \mapsto u \otimes 1^{\sigma}=u \otimes 1 \mapsto u \in \mathrm{H}^{0}\left(\mathbb{P}^{1}, \mathcal{L}_{2}\right) .
$$

On the other hand,

$$
x \otimes y=1 \otimes u \mapsto 1 \otimes u^{\sigma}=1 \otimes(u+1) \mapsto u+1 \in \mathrm{H}^{0}\left(\mathbb{P}^{1}, \mathcal{L}_{2}\right) .
$$


Since $x \otimes x \mapsto 1 \in \mathrm{H}^{0}\left(\mathbb{P}^{1}, \mathcal{L}_{2}\right)$, we see that we have the relation $x y=y x+x^{2}$, in $B\left(\mathbb{P}^{1}, \mathcal{L}, \sigma\right)$. As one might expect, a little more work shows that

$$
B\left(\mathbb{P}^{1}, \mathcal{L}, \sigma\right) \cong k\{x, y\} /\left(x y-y x-x^{2}\right) .
$$

We leave it as an exercise for the reader to show that, if one uses the automorphism $\sigma: u \mapsto \lambda u$, for some $\lambda \in k^{*}$, then $B\left(\mathbb{P}^{1}, \mathcal{O}_{\mathbb{P}^{1}}(1), \sigma\right)$ is isomorphic to the other quantum plane $k\{x, y\} /(x y-\lambda y x)$.

\section{Algebras With linear growth}

As was pointed out in the introduction, it is necessary to have some type of dimension function on noncommutative rings. There is a multitude of such functions in the noncommutative case, each with its own advantages and disadvantages. The interested reader may look in [40], 48, [96, 97] for some relevant references.

As an example of a reasonably well-behaved invariant we will now introduce Gelfand-Kirillov dimension, which basically measures the growth of a ring. Fix a finitely generated $k$-algebra $R$. Pick generators $r_{1}, \ldots, r_{m}$ for $R$ and define an increasing filtration on $R$ by $\Gamma_{1}=k+\sum_{1}^{m} r_{i} k$ and $\Gamma_{j}=\Gamma_{1}^{j}$ for $j>1$. Then the Gelfand-Kirillov dimension of $R$ is defined to be

$$
\begin{aligned}
\operatorname{GKdim}(R) & =\inf \left\{\alpha \in \mathbb{R}: \operatorname{dim}_{k} \Gamma_{n} \leq n^{\alpha} \text { for all } n \gg 0\right\} \\
& =\limsup \frac{\ln \left(\operatorname{dim} \Gamma_{n}\right)}{\ln (n)} .
\end{aligned}
$$

It is routine to check that $\mathrm{GKdim}(R)$ is independent of the choice of generators. Also, for a finitely generated commutative algebra $A, \operatorname{GKdim}(A)$ reduces to the degree of the Hilbert-Samuel polynomial as defined, for example, in [11, Chapter 11], and so $\operatorname{GKdim}(A)$ equals the Krull dimension of $A$. For these and other basic facts about this dimension, the reader is referred to [40].

As so often happens, many of the basic properties of Gelfand-Kirillov dimension are much nastier in the noncommutative universe than in the commutative one. For example, it need not be an integer; indeed there are examples of Bergman that show that $\operatorname{GKdim}(R)$ can take any real number $\alpha \geq 2$ [40, Theorem 2.9]. Fortunately, if $\operatorname{GKdim}(R) \leq 2$, then $\operatorname{GKdim}(R)$ equals zero, one or two. (See [40, Theorems 2.5, 2.9 and Proposition 1.4].) Curiously, though, there are no examples of domains with non-integer Gelfand-Kirillov dimension.

If $\operatorname{GKdim}(R)=0$, it is an easy exercise to show that $R$ is a finite dimensional $k$-algebra. Thus the first interesting case occurs when $\operatorname{GKdim}(R)=1$.

Proposition 4.1. 70 Let $R$ be a domain, with $\operatorname{GKdim}(R)=1$, that is finitely generated as a $k$-algebra. Then $R$ is commutative.

If one takes a geometric point of view, whereby an (ungraded) noncommutative domain of Gelfand-Kirillov dimension $n$ should be regarded as the coordinate ring of a noncommutative irreducible affine scheme of dimension $n$, then this result says that such a scheme is a genuine irreducible curve. Similarly, an irreducible noncommutative projective scheme of dimension zero, which would correspond to a graded domain $R$ with $\operatorname{GKdim}(R)=1$, is just a point. Almost nothing is known about higher dimensional affine analogues of this proposition, but the underlying thesis of this paper is that the projective analogues do seem to exist. 
Proposition 4.1 actually generalizes to any ring of Gelfand-Kirillov dimension one, even over fields that are not algebraically closed. The notation is explained in \$1.1.

Proposition 4.2. 69] Let $R$ be a finitely generated $k$-algebra and assume that $\operatorname{GKdim}(R)=1$. Then $N(R)$ is nilpotent and $\bar{R}=R / N(R)$ is a finite module over its centre $Z=Z(\bar{R})$. Moreover, $Z$ is a finitely generated semiprime $k$-algebra of Krull dimension one. In particular, $Z$ and $\bar{R}$ are noetherian.

The reason why Proposition 4.1 follows from Proposition 4.2 is as follows: If $R$ is a domain, then $Z=Z(R)$ is also domain, and its field of fractions $F=\operatorname{Frac}(Z)$ has transcendence degree at most one. Since $k$ is algebraically closed, Tsen's Theorem 21. Exercise 7, p. 375] says that $F$ is the only division ring, finite dimensional as an $F$-module, with centre $F$. Since $R F$ is just such a division ring, $R$ is commutative and we are done.

\section{Noncommutative Projective Curves I: Domains of Quadratic GROWTH}

Having dismissed the algebras of Gelfand-Kirillov dimension one, the next case of interest is that of algebras of Gelfand-Kirillov dimension two. Here, we are concerned with the structure of finitely graded algebras, since these would provide the homogeneous coordinate rings of the (putative) noncommutative projective curves.

In this section, we describe all finitely graded domains of Gelfand-Kirillov dimension 2, together with their projective geometry. The case of prime rings over arbitrary fields will be covered in $\sqrt[6]{6}$. Roughly speaking, these results show that there are only two types of noetherian domains $R$ that can occur: one either has a twisted homogeneous coordinate ring $B(Y, \mathcal{L}, \sigma)$, in the sense of 43 , or one has an analogue of the characteristic zero version of the ring $S=k+U y$ from (2.3). In either case, $\mathrm{QGr}(R)$ is essentially $\mathrm{Q} \operatorname{ch}(Y)$, for some curve $Y$. Thus these results really do justify the intuition that any such domain is the homogeneous coordinate ring of a noncommutative projective curve.

Fix a finitely graded domain $R$ with $\operatorname{GKdim}(R)=2$. As in commutative algebra, a natural first step towards describing $R$ is to determine its "birational" structure, that is, to determine the graded ring of fractions $Q=Q(R)$. This is the ring obtained by inverting the homogeneous elements in $R$ (for the nontrivial proof that this ring even exists combine [50, Theorem C.I.1.6] with [40, Theorem 4.12]). Then [50, Theorems A.I.5.8 and C.I.1.6] show that $Q(R)=D\left[z, z^{-1} ; \sigma\right]$, where $\sigma$ is an automorphism of the division ring $D$ and $D\left[z, z^{-1} ; \sigma\right]$ is the twisted Laurent polynomial ring; thus, as a $k$-vector space, it is isomorphic to the usual Laurent polynomial ring, but multiplication is twisted by $z a=\sigma(a) z$, for $a \in D$. However, much more is true:

Theorem 5.1. 4, Theorem 0.1] Assume that $R$ is a finitely graded domain with $\operatorname{GKdim}(R)=2$ and write $Q(R)=D\left[z, z^{-1} ; \sigma\right]$ for the graded quotient ring of $R$.

(i) $D=K$ is a field that is finitely generated and of transcendence degree one as an extension of $k$.

(ii) Conversely, if $Q=Q(R)$ is as in (i) and if $S$ is any finitely graded subring of $Q$, then $\operatorname{GKdim}(S) \leq 2$. Indeed, there even exists a constant $c$ such that $\operatorname{dim}_{k} S_{n} \leq$ cn, for all $n \geq 1$. 
The idea behind the proof is to show that $\operatorname{GKdim}(T) \leq 1$, for any finitely generated subalgebra $T \subseteq D$. Most of the theorem then follows from Proposition 4.1.

The notation of Theorem 5.1 will be fixed for the rest of the section. By the theory of algebraic curves [26, $\S$ I.6], $K=Q(R)_{0}$ is the function field of a unique smooth projective curve $X$ and the automorphism $\sigma$ induces an automorphism, which will still be denoted by $\sigma$, on $X$. Thus the theorem can really be regarded as saying that $R$ is determined birationally by an automorphism of that curve.

However we can give a much more detailed geometric description of the ring $R$ and the model for our program is again provided by algebraic geometry, in the form of Serre's Theorem 2.1.2. Remarkably, this generalizes completely:

Theorem 5.2. [4, Theorem 0.2] Let $R$ be a connected graded domain of GKdimension 2 and assume that $R$ is generated by elements of degree 1 . Then there exist:

(i) A projective curve $Y$, birational to $X$ and with an induced action of $\sigma$,

(ii) An ample invertible sheaf $\mathcal{L}$ on $Y$,

such that $R$ embeds into the twisted homogeneous coordinate ring $B=B(Y, \mathcal{L}, \sigma)$ with $\operatorname{dim}_{k}(B / R)<\infty$. Equivalently, $R_{n} \cong \mathrm{H}^{0}\left(Y, \mathcal{L} \otimes \mathcal{L}^{\sigma} \otimes \cdots \otimes \mathcal{L}^{\sigma^{n-1}}\right)$ for $n \gg 0$.

Since ample invertible sheaves on curves are automatically $\sigma$-ample, we may immediately apply Theorem 3.2 to obtain:

Corollary 5.3. Let $R$ be as in Theorem 5.2. Then $R$ is a noetherian domain, and the quotient category $\operatorname{qgr}(R)$ is equivalent to the category $\operatorname{coh}(Y)$ of coherent sheaves on $Y$.

Since twisted homogeneous coordinate rings are so well understood, one can use the theorem to give further results about the structure of $R$. For example, under the equivalence of the corollary, two-sided ideals of $R$ correspond to the (ideals of definition of) closed $\sigma$-invariant subschemes of $Y$. This quickly leads to:

Corollary 5.4. Let $R$ be as in Theorem 5.2.

(i) If the order $|\sigma|$ of $\sigma$ is finite, then $R$ is a finite module over its centre.

(ii) If $|\sigma|=\infty$, then $X$ is either rational or elliptic. In either case, $R$ is a primitive ring; that is, $R$ has a faithful simple right module.

(iii) If $X$ is rational and $|\sigma|=\infty$, then $R$ has just one or two graded prime ideals $P_{i}$ of height one and, in each case, $R / P_{i}$ is a domain. All other non-zero prime ideals $Q$ of $R$ are of finite codimension in $R$.

(iv) If $X$ is elliptic and $|\sigma|=\infty$, then $Y=X$ and the only non-zero prime ideal of $R$ is the augmentation ideal $R_{+}=\bigoplus_{i>0} R_{i}$.

The case that $R$ is not generated in degree one, however, differs essentially from commutative algebra and exhibits a striking new phenomenon.

To see this, define the $d$-th Veronese ring of $R$ to be the graded ring $R^{(d)}=$ $\bigoplus_{n \geq 0} R_{n d}$, graded by $R_{n}^{(d)}=R_{n d}$. If $R$ is generated in degree one, then it is easy to prove that $\mathrm{Q} \operatorname{Gr}(R) \cong \mathrm{Q} \operatorname{Gr}\left(R^{(d)}\right)$. So from a geometrical standpoint $R$ and $R^{(d)}$ are equivalent. Even if $R$ is not generated in degree one, then it may happen, as is always the case if $R$ is commutative, that some Veronese ring $R^{(d)}$ is generated in degree one. If so, then $R$ is a finite $R^{(d)}$-module and, as in the commutative case, the structure of $R$ is closely related to that of $R^{(d)}$. Theorem 5.2 can be applied 
to such rings and, after the appropriate modifications, analogues of Corollaries 5.3 and 5.4 will hold for $R$. The details can be found in [4, §6].

Unlike the commutative case, though, it may also happen that no Veronese ring is generated in degree one; indeed, Theorem 5.6(i) can be regarded as saying that this is the generic case when $|\sigma|=\infty$. An explicit example is given, over any field $k$, by the ring $S=k+U y$ from (2.3) (see 76. Corollary 3.2]). The next two theorems from [4] summarize the main results about this class of rings and, in particular, explain the dichotomy (2.4) that $S=k+U y$ is noetherian if and only if char $k=0$.

Theorem 5.5. [4, Theorem 0.4] Assume that $R$ is a finitely graded domain of GK-dimension 2.

(i) If $|\sigma|=\infty$, then $R$ is a noetherian primitive ring, and every Veronese ring of $R$ is finitely generated.

(ii) Assume that $|\sigma|=d<\infty$ and that no Veronese ring of $R$ is generated in degree one. Then $R$ is not noetherian. Indeed, $R^{(d)}$ is a commutative ring which is not finitely generated.

Part (ii) of the theorem is easy: Since $\sigma^{d}=1, R^{(d)}$ is a subring of the commutative ring $K\left[z^{d}, z^{-d}\right]$, and so is commutative. If $R^{(d)}$ were finitely generated, then classical results ensure that some further Veronese ring is generated in degree one. Thus the interesting case of the theorem is when $|\sigma|=\infty$. In this case, much more can be said about the structure of $R$, but for simplicity we only state the result for a Veronese. A complete description of the rings which arise in case (ii) of Theorem 5.5 remains to be found.

Theorem 5.6. [4, Theorem 0.5] Let $R$ be as in Theorem [5.5. Assume that no Veronese ring of $R$ is generated in degree one and that $|\sigma|=\infty$. Then, after replacing $R$ by an appropriate Veronese ring, the following hold:

(i) There is an algebraic curve $Y$, birational to $X$ and with an induced action of $\sigma$, and two invertible sheaves $\mathcal{L} \subsetneq \mathcal{N}$ on $Y$, such that

$$
R_{m}=\mathrm{H}^{0}\left(Y, \mathcal{L} \otimes \mathcal{N}^{\sigma} \otimes \cdots \otimes \mathcal{N}^{\sigma^{m-1}}\right), \quad \text { for all } m \geq 1 .
$$

Moreover, $\mathcal{L}=\mathcal{N}$ locally on every finite orbit of $\sigma$.

(ii) $R$ is generated in degrees one and two.

(iii) $R$ is contained in the twisted homogeneous coordinate ring $B=B(Y, \mathcal{N}, \sigma)$. Moreover, $B$ is finitely generated as a right $R$-module but infinitely generated as a left $R$-module.

(iv) $R$ does not satisfy $\chi_{1}$. However, $\operatorname{qgr}(R)$ is equivalent to $\operatorname{coh}(Y)$.

The converse to part (i) holds in the sense that, if $\mathcal{L}$ and $\mathcal{N}$ are as defined there, then $R=k \oplus \bigoplus_{n \geq 1} \mathrm{H}^{0}\left(Y, \mathcal{L} \otimes \mathcal{N}^{\sigma} \otimes \cdots \otimes \mathcal{N}^{\sigma^{n-1}}\right)$ is a noetherian connected graded domain. Moreover, if $\mathcal{L} \neq \mathcal{N}$, then no Veronese ring $R^{(d)}$ is generated in degree one.

Exercise. Write the ring $S=k+U y$ from (2.3) in the form described by Theorem 5.6. Under the appropriate identifications, you should find that $\mathcal{L}=\mathcal{O}_{\mathbb{P}^{1}} \subset$ $\mathcal{N}=\mathcal{O}_{\mathbb{P}^{1}}(1)$ and that $B(Y, \mathcal{N}, \sigma)=y U y^{-1} \cong U$.

One curious consequence of the results from this section is that they contradict the pervasive feeling that noncommutative rings are more varied and complicated than their commutative counterparts, notably that one should expect that the 
rings that are farthest from commutative should have the worst properties. For connected graded domains of dimension two, the opposite is true: The only nasty (non-noetherian) examples are close to commutative (formally, they embed into a finite free module over a commutative subalgebra), while any such domain that is not close to commutative is defined in terms of a curve of genus $\leq 1$.

Let us at least indicate how to construct the curve $Y$ in Theorems 5.2 and 5.6 We will only do this in the case when $R$ is generated by $R_{1}$, but the general case is similar. Fix a non-zero element $z \in R_{1}$, and write $R_{m}=\mathcal{R}_{m} z^{m}$, so that $\mathcal{R}_{m}$ is a subspace of the function field $Q(R)_{0}=k(X)$. Multiplication in $R$ translates to the statement that

$$
\mathcal{R}_{n} \sigma^{n}\left(\mathcal{R}_{m}\right) \subseteq \mathcal{R}_{n+m} \quad \text { for } n, m \geq 0 .
$$

The space $\mathcal{R}_{m}$ defines a morphism $\pi_{m}: X \rightarrow \mathbb{P}\left(\mathcal{R}_{m}^{*}\right)$ [26, Theorem II.7.1], and we set $Y_{m}=\operatorname{Im}\left(\pi_{m}\right)$. A key step in the proof is to show, possibly after replacing $R$ by a Veronese ring $R^{(d)}$, that $Y_{m} \cong Y_{m+1}$ for all $m \gg 0$. Once this has been achieved, then one simply takes $Y=Y_{m}$, for some large $m$. Set $\mathcal{L}_{m}=\mathcal{O}_{Y} \mathcal{R}_{m}$, which is an invertible sheaf on $Y$. A second key fact is that

$$
\mathcal{R}_{m}=\mathrm{H}^{0}\left(Y, \mathcal{L}_{m}\right) \quad \text { for all } m \gg 0 .
$$

We are now done. Indeed, since $R$ is generated by $R_{1}$, (5.1) implies that $\mathcal{R}_{m}=$ $\mathcal{R}_{1} \mathcal{R}_{1}^{\sigma} \cdots \mathcal{R}_{1}^{\sigma^{m-1}}$ and so $\mathcal{L}_{m}=\mathcal{L}_{1} \otimes \cdots \otimes \mathcal{L}_{1}^{\sigma^{m-1}}$. Thus Theorem 5.2 holds with $\mathcal{L}=\mathcal{L}_{1}$.

\section{Noncommutative Projective Curves II: Prime Rings}

In this section, we outline how to generalize the results of the last section to prime rings of quadratic growth. There are two reasons for this. Despite the fact that most of the results in this survey are concerned with domains, the correct context is probably that of prime rings - certainly this is true for the most significant applications of ring theory - and so this section can be thought of as illustrating how such generalizations might go. Secondly, one of the best understood areas of noncommutative algebra is that of classical orders (which we define to be nongraded prime rings of Gelfand-Kirillov dimension one). Given the success of $\S 5$. one should expect a similar generalization of the theory of classical orders to the projective setting and this section provides just such a theory. Once one works with prime rings rather than domains, the standing assumption that $k$ be algebraically closed provides no advantages and so the results of this section actually hold for arbitrary base fields. All the results of this section are taken from [5].

Thus, we wish to study finitely graded prime $\operatorname{rings} R$ with $\operatorname{GKdim}(R)=2$. Although the results of the last section generalize naturally, there are some differences and to explain this we begin with a series of examples. In all these examples, we begin with the standard quantum plane $U=\mathbb{C}\{x, y\} /\left(x y-y x-x^{2}\right) \cong B\left(\mathbb{P}^{1}, \mathcal{O}(1), \sigma\right)$, as in (3.4), but we now consider subrings of the $2 \times 2$ matrix ring $V=M_{2}(U)$.

The first obvious difference is that we have to use, as our basic building block, rings $B\left(\mathcal{E}, \mathcal{B}_{1}, \tau\right)$ defined in terms of orders $\mathcal{E}$ over a curve $Y$ rather than the twisted homogeneous coordinate rings $B(Y, \mathcal{L}, \sigma)$ that were used in $\$ 5$. This does not cause any serious complications; in fact it is even an advantage since it allows for more subtle examples from the same basic construction. The construction is given in detail in [5], but the idea is as follows. Assume that $X$ is a projective variety and that $\mathcal{E}$ is an order inside a central semisimple $k(X)$-algebra $A$. Let $\tau$ be a 
$k$-algebra automorphism of $A$ that restricts to an automorphism $\sigma$ of $k(X)$ and $X$ and assume that $\mathcal{L}$ is an invertible sheaf of $\left(\mathcal{E}, \mathcal{E}^{\tau}\right)$-bimodules contained in $A$. Modulo these definitions, the twisting ring $B=B(\mathcal{E}, \mathcal{L}, \tau)$ looks just like the twisted homogeneous coordinate ring $B(X, \mathcal{L}, \sigma)$. Indeed,

$$
B \cong \bigoplus_{n} \mathrm{H}^{0}\left(X, \mathcal{L}_{n}\right) \quad \text { where } \quad \mathcal{L}_{n}=\mathcal{L} \otimes_{\mathcal{E}^{\tau}} \mathcal{L}^{\tau} \otimes_{\mathcal{E}^{\tau^{2}}} \cdots \otimes_{\mathcal{E}^{\tau^{n-1}}} \mathcal{L}^{\tau^{n-1}}
$$

and multiplication is defined by the analogue of (3.2). If $\mathcal{L}$ is ample as an $\mathcal{O}_{X^{-}}$ module, then $s=\tau_{*}\left(-\otimes_{\mathcal{E}} \mathcal{L}\right)$ is ample in the sense of Definition 2.1.4, and so Theorem 2.1.5 can again be used to describe $B$.

As an exercise, the reader may like to prove that the matrix ring $V=M_{2}(U)$ satisfies $V \cong B\left(\mathcal{E}, \mathcal{B}_{1}, \tau\right)$, where $\mathcal{E}=M_{2}\left(\mathcal{O}_{X}\right), \mathcal{B}_{1}=\mathcal{O}_{X}(1) \otimes_{\mathcal{O}_{X}} \mathcal{E}$ and $\tau$ is the natural extension to $\mathcal{E}$ of the automorphism $\sigma$ from (3.4). A more subtle example appears at the end of the section.

A more serious complication occurs if one tries to generalize Theorem 5.2 to the prime case, since it is now easy to construct rings generated in degree one that are not noetherian, let alone twisting rings. Here are a couple of simple examples.

Example 6.1. Consider

$$
R^{\prime}=\left(\begin{array}{lr}
U & U x \\
U & \mathbb{C}+U x
\end{array}\right)
$$

Then it is not hard to show 76 that $R^{\prime}$ is neither left nor right noetherian. However, it is generated in degree one. In fact, this example still works if we replace $U$ by the commutative polynomial ring $\mathbb{C}[x, y]$.

Example 6.2. Consider

$$
R^{\prime \prime}=\left(\begin{array}{lr}
U & U y \\
U & \mathbb{C}+U y
\end{array}\right)
$$

Then $R^{\prime \prime}$ is both left and right noetherian and it is still generated in degree one (see [76], again). However, it follows from (6.1) below that $R^{\prime \prime}$ is not a twisting ring.

Thus generation in degree one is not very helpful and so one needs more subtle geometric conditions to determine the twisting rings, or even when a ring is noetherian. These are provided by (6.1) and Theorem 6.6. Observe that $R^{\prime}=\mathbb{C}+I^{\prime}$, and $R^{\prime \prime}=\mathbb{C}+I^{\prime \prime}$, for left ideals $I^{\prime}$ and $I^{\prime \prime}$ of $V$. The difference between them is that $V / I^{\prime}$ is supported (in a manner that is made precise in Theorem 6.3 ) in $\mathbb{P}^{1}$ at the fixed point of $\sigma$, whereas $V / I^{\prime \prime}$ is supported on an infinite orbit of $\sigma$. Thus $R^{\prime \prime}$ looks very like - and behaves very like - the ring $\mathbb{C}+U y$ that played such a prominent rôle in the last section. In contrast, the analogue to $R^{\prime}$ would be $\mathbb{C}+U x$, which is not finitely generated and so never appeared in $\$ 5$.

With these examples in hand, the main result of this section is easy to state informally: A finitely graded prime ring $R$, with $\operatorname{GKdim}(R)=2$, is noetherian if and only if some Veronese ring $S=R^{(d)}$ is either a twisting ring $B=B\left(\mathcal{E}, \mathcal{B}_{1}, \tau\right)$ or else of the form $S=k+I$, where $I$ is a left ideal of $B$ such that $B / I$ is supported on a union of infinite orbits of $\sigma$. In either case, $\operatorname{qgr}(S) \simeq \operatorname{coh}(\mathcal{E})$.

We now formalize these ideas. It is actually easier to work with semiprime rather than prime rings, so fix a base field $k$ and let $S=\bigoplus_{i \geq 0} S_{i}$ be a semiprime finitely graded $k$-algebra with quadratic growth. Unlike the last section, the assertion that the quotient ring $Q(S)$ exists is a genuine assumption and is one of the equivalent 
definitions that $S$ be a Goldie ring (see [50, Theorem C.I.1.6] for the details and note that semiprime noetherian rings are automatically Goldie). For simplicity, we will make two further assumptions: Define $S$ to be nice if

(a) $\operatorname{GKdim}(S / P)=2$ for all minimal prime ideals $P$ of $S$ and

(b) $S$ contains a non-zero divisor $z \in S_{1}$.

These assumptions are only being made to simplify our notation and can be easily removed (see [5]). Indeed, (a) will always hold if $S$ is prime, while (b) can always be assumed by passing to a Veronese ring.

One advantage of condition (b) is that the graded quotient ring $Q=Q(S)$ of $S$ can now be written $Q=A\left[z, z^{-1} ; \tau\right]$, where the semisimple Artinian algebra $A$ equals $Q_{0}$ and $\tau$ is an automorphism of $A$ [50, Lemma A.II.1.5]. Standard techniques from Goldie's theory (see [3, §1]) allow one to generalize Theorem 5.1 to this context and they show that $A$ is a finite module over its centre $K$ and $K$ is a direct sum of fields of transcendence degree one. Let $X$ denote the smooth projective model of $K / k$ and write $\sigma$ for the restriction of $\tau$ to $K$ and $X$. We fix this notation throughout this section.

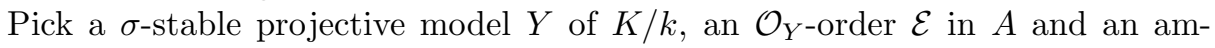
ple invertible $\left(\mathcal{E}, \mathcal{E}^{\tau}\right)$-bimodule $\mathcal{B}_{1}$. The next result provides the general version of Example 6.2 and can also be thought of as the natural generalization of the construction of Theorem 5.6 (i).

Theorem 6.3. Let $Y, \mathcal{E}$ and $\mathcal{B}_{1}$ be as above. Let $\mathcal{R}_{1}$ be an essential right $\mathcal{E}^{\tau}$ submodule of $\mathcal{B}_{1}$ such that $1 \in \mathrm{H}^{0}\left(Y, \mathcal{R}_{1}\right)$ and such that $\mathcal{R}_{1}=\mathcal{B}_{1}$ locally on every finite orbit of $\sigma$ on $Y$. For $n \geq 1$, set

$$
\mathcal{R}_{n}=\mathcal{R}_{1} \otimes_{\mathcal{E}^{\tau}} \mathcal{B}_{1}^{\tau} \otimes_{\mathcal{E}^{\tau^{2}}} \cdots \otimes_{\mathcal{E}^{\tau^{n-1}}} \mathcal{B}_{1}^{\tau^{(n-1)}}
$$

Then $R=k+\bigoplus_{n \geq 1} \mathrm{H}^{0}\left(Y, \mathcal{R}_{n}\right)$ is a noetherian nice algebra.

Exercise. Write the ring $R^{\prime \prime}$ from Example6.2 in this form. The answer, modulo a left-right switch, can be found in [5, Example 5.13].

Once again, the rings constructed by Theorem 6.3 have a pleasant module structure.

Corollary 6.4. Let $R$ be the ring constructed by Theorem 6.3 Then

(i) The categories $\operatorname{qgr}(R)$ and $\operatorname{coh}(\mathcal{E})$ are equivalent.

(ii) $R$ is a subidealizer in $B=B\left(\mathcal{E}, \mathcal{B}_{1}, \tau\right)$ in the sense that, for some $r \geq 1$, $V=\bigoplus_{i \geq r} R_{i}$ is a right ideal of $B$. Moreover, $B$ will be a finitely generated right $R$-module, although it may be infinitely generated as a left $R$-module.

The main result of $[5]$ is the following partial converse to Theorem 6.3 ,

Theorem 6.5. Let $S$ be a noetherian nice algebra. For some n, the Veronese ring $R=S^{(n)}$ has the form described in Theorem 6.3. Moreover, if $\mathcal{S}_{n}=\mathcal{O}_{Y} S_{n}$, then $S_{n}=\mathrm{H}^{0}\left(Y, \mathcal{S}_{n}\right)$ for all $n \gg 0$.

If $S$ is a noetherian nice algebra, as in Theorem 6.5, then the module structure of $S$ may be more complex than that described in Corollary 6.4-but then this is also true for a commutative ring that is not generated in degree one. Nevertheless, the equivalence nearly extends to this case. Formally, by [4 Proposition 6.1(iv)] the category of graded 1-critical $S$-modules that are generated in degrees $n \mathbb{Z}$ is equivalent to the category of 1-critical modules over $R=S^{(n)}$ and so one may 
apply Corollary 6.4 via this correspondence. Consequently, the simple objects in $\operatorname{qgr}(S)$ that are generated in degrees $n \mathbb{Z}$ are in one-to-one correspondence with the simple $\mathcal{E}$-modules.

The final question we need to address is to determine geometrically when a nice algebra is noetherian. To describe this condition, it is convenient to write a nice algebra $R$ as $R=\bigoplus \mathcal{R}_{i} z^{i}$, so that $\mathcal{R}_{i} \subset A$ for all $i$. The fact that $R$ is a graded ring translates to the inclusions $\mathcal{R}_{i} \mathcal{R}_{j}^{\tau^{i}} \subseteq \mathcal{R}_{i+j}$. Let $N$ denote the reduced norm map $A \rightarrow K$. For a point $p \in X$ and a subset $V$ of $A$, we denote by $\mathcal{P}(V ; p)$ the maximum order of a pole at $p$ of $N(v)$, for $v \in V$. The key condition is

$$
\mathcal{P}\left(\mathcal{R}_{i} ; p\right)+\mathcal{P}\left(\mathcal{R}_{j}^{\tau^{i}} ; p\right)=\mathcal{P}\left(\mathcal{R}_{i+j} ; p\right) .
$$

If $A=K$, then (6.1) will hold whenever $\mathcal{R}_{i} \mathcal{R}_{j}^{\tau^{i}}=\mathcal{R}_{i+j}$. However, this is not true in general - which should come as no surprise, since it is related to the fact that the determinant of a sum (of products) of matrices cannot be described in terms of the determinants of the individual matrices. In particular, it fails for the rings $R^{\prime}$ and $R^{\prime \prime}$ of Examples 6.1 and 6.2. If one computes $\mathcal{P}\left(\mathcal{R}_{i} ; p\right)$ for $R^{\prime}$ and $R^{\prime \prime}$, one finds that (6.1) fails for $R^{\prime}$ at just one point, the fixed point of $\sigma$, whereas for $R^{\prime \prime}$ it fails only on an infinite orbit of $\sigma$. The details can be found in [5, Example 5.14]. Thus the following result should come as no surprise.

Theorem 6.6. Let $S$ be a nice algebra. Then:

(i) $S$ is right noetherian if and only if it is left noetherian. This is true if and only if there is an integer $n$ such that $R=S^{(n)}$ is a nice algebra for which (6.1) holds on all finite orbits of $\sigma$ on $X$.

(ii) Some Veronese ring $S^{(d)}$ is a twisting ring $B\left(\mathcal{E}, \mathcal{B}_{1}, \tau\right)$ if and only if some $R=S^{(n)}$ is a nice algebra for which (6.1) holds at all $p \in X$.

Many of the standard examples in PI theory can be modified to be graded rings of Gelfand-Kirillov dimension two, and so can be reinterpreted in terms of twisting rings. As an example, we invite the reader to describe the following algebra as a twisting ring:

$$
B=\left\{\left(\begin{array}{rr}
a+y b_{11} & x \frac{\partial(a)}{\partial x}+y b_{12} \\
y b_{21} & a+y b_{22}
\end{array}\right): a, b_{i j} \in k[x, y]\right\} .
$$

One solution is $B \cong B\left(\mathcal{E}, \mathcal{B}_{1}, \tau\right)$, where $\mathcal{E}=\mathcal{O}_{\mathbb{P}^{1}}+M_{2}(\mathcal{O}(-1))$ and $\mathcal{B}_{1}=\mathcal{E} \otimes_{\mathcal{O}} \mathcal{O}(1)$. One interesting feature of this example is that $\tau$ is the inner automorphism given by conjugation by $\left(\begin{array}{ll}1 & 1 \\ 0 & 1\end{array}\right)$. The details can be found in [5, Example 5.14]. This is one of the standard examples of a noetherian ring that satisfies a polynomial identity but is not a finite module over its centre [62, Example 5.1.16].

\section{Noncommutative Smooth PRoper CURVES}

Although the results from the last two sections are very satisfying they should be only part of the story. As in the commutative case one would very much prefer to define noncommutative curves abstractly and then to show that they can indeed be described by graded rings. We provide one possible method in this section.

This has three steps: first, we describe the homological properties that are satisfied by a smooth proper curve and which should be satisfied by any noncommutative analogue of such a curve. Secondly, we provide a list of abelian categories that satisfy these conditions. Finally, we show that these categories are indeed determined by those properties. 
7.1. Geometric conditions on abelian categories. In this subsection, we assume that $X$ is a smooth, proper and connected $k$-scheme of dimension $n$ and we isolate the homological conditions that we want to be satisfied by our noncommutative analogues of $\mathcal{C}=\operatorname{coh}(X)$. For a discussion of the singular case we refer to 7.4. One could also have associated $\mathrm{Qch}(X)$ to $X$ but, as Theorem 1.1.1 shows, this would not make too much difference.

The category $\mathcal{C}=\operatorname{coh}(X)$ clearly satisfies the following conditions.

(C1) $\mathcal{C}$ is noetherian.

(C2) $\mathcal{C}$ is Ext-finite. In other words, the Yoneda Ext group satisfies $\operatorname{Ext}^{i}(A, B)<\infty$ for all $A, B \in \mathcal{C}$ and all $i$.

(C3) $\mathcal{C}$ has homological dimension $n$ in the sense that $\operatorname{Ext}^{i}(A, B)=0$ for $A, B \in \mathcal{C}$ and for $i>n$, and $n$ is minimal with this property.

Unfortunately, far too many noncommutative rings have finite homological dimension and so these three conditions are not nearly sufficient. A fundamental, but slightly more subtle, property of smooth proper schemes is the Serre duality theorem. This asserts that there exists a dualizing sheaf $\omega$ such that for $\mathcal{F} \in \mathcal{C}$ there are natural isomorphisms

$$
H^{i}(X, \mathcal{F}) \cong \operatorname{Ext}^{n-i}(\mathcal{F}, \omega)^{*} .
$$

A very elegant reformulation of Serre duality was given in [17]. Let $D^{b}(\mathcal{G})$ denote the derived category of bounded complexes over an abelian category $\mathcal{G}$. A Serre functor on $D^{b}(\mathcal{G})$ is an autoequivalence $F: D^{b}(\mathcal{G}) \rightarrow D^{b}(\mathcal{G})$ such that there are isomorphisms

$$
\operatorname{Hom}(A, B) \cong \operatorname{Hom}(B, F A)^{*}
$$

which are natural in $A, B \in D^{b}(\mathcal{G})$. Then Bondal and Kapranov show that Serre duality can be reinterpreted as saying:

$(\mathrm{C4}) \mathcal{C}$ satisfies Serre duality in the sense that there exists a Serre functor on $D^{b}(\mathcal{C})$.

7.2. Some examples. An obvious test question for noncommutative geometry would be to to classify abelian categories satisfying the homological properties (C1)(C4) listed above. Since this is far too optimistic a project at present, we just consider the hereditary categories, that is the ones of homological dimension one. Here, at least one has a chance, since there is a small list of examples.

The most obvious hereditary noetherian k-linear Ext-finite abelian categories satisfying Serre duality are:

(E1) Let $X$ be a smooth projective connected curve over $k$ with function field $K$ and let $\mathcal{O}$ be a sheaf of hereditary $\mathcal{O}_{X}$ orders in $M_{n}(K)$ (see [56]). Then one proves exactly as in the commutative case that $\mathcal{C}_{1}=\operatorname{coh}(\mathcal{O})$ satisfies Serre duality. The Serre functor is given by tensoring with $\mathcal{H o m}\left(\mathcal{O}, \omega_{X}\right)$ [1].

Three rather special examples, for which it is easy to check $(\mathrm{C} 1)-(\mathrm{C} 4)$, are:

(E2) $\mathcal{C}_{2}$ consists of the finite dimensional nilpotent representations of the quiver $\tilde{A}_{n}$ [59] (possibly with $n=\infty$ ) with all arrows oriented in the same direction. The Serre functor is given by rotating one place in the direction of the arrows and shifting one place to the left in the derived category.

(E3) $\mathcal{C}_{3}=\operatorname{gr}_{\mathbb{Z}^{2}}(k[x, y]) /($ tor $)$ consists of the finitely generated $\mathbb{Z}^{2}$-graded $k[x, y]$ modules modulo the finite dimensional modules. The Serre functor is given by the shift $M \mapsto M(-1,-1)[\mathbb{1}]$ 
(E4) The category $\mathcal{C}_{3}$ has a natural automorphism $\sigma$ of order two which sends a graded module $\left(M_{i j}\right)_{i j}$ to $\left(M_{j i}\right)_{i j}$ and which exchanges the $x$ and $y$ action. Let $\mathcal{C}_{4}$ be the category of $\mathbb{Z} / 2 \mathbb{Z}$ equivariant objects in $\mathcal{C}$, i.e. pairs $(M, \phi)$ where $\phi$ is an isomorphism $M \rightarrow \sigma(M)$ satisfying $\sigma(\phi) \phi=\operatorname{id}_{M}$. Properties (C1) $-(\mathrm{C} 4)$ follow easily from the fact that they hold for $\mathcal{C}_{3}$. In particular the Serre functor is obtained from the one on $\mathcal{C}_{3}$ in the obvious way. As described, this construction requires char $k \neq 2$, but this can be circumvented [57].

We now come to the most subtle example.

(E5) Let $Q$ be a connected locally finite quiver such that the opposite quiver has no infinite oriented paths. For a vertex $x \in Q$ we have a corresponding projective representation $P_{x}$ and an injective representation $I_{x}$ and by our hypotheses $I_{x}$ is finite dimensional. Let $\operatorname{rep}(Q)$ be the finitely presented representations of $Q$. The category of all representations of $Q$ is hereditary and hence $\operatorname{rep}(Q)$ is an abelian category. It is easy to check that $I_{x} \in \operatorname{rep}(Q)$. Hence the functor $P_{x} \mapsto I_{x}$ may be derived to yield an endofunctor $F: D^{b}(\operatorname{rep}(Q)) \rightarrow$ $D^{b}(\operatorname{rep}(Q))$. This functor $F$ behaves like a Serre functor in the sense that we have natural isomorphisms as in (7.1), but unfortunately $F$ need not be an autoequivalence. However, there is a formal procedure to invert $F$ so as to obtain a true Serre functor [57]. This yields a hereditary category $\widetilde{\operatorname{rep}}(Q)$ which satisfies Serre duality. Under a technical additional hypothesis (see [57]) $\mathcal{C}_{5}=\widetilde{\operatorname{rep}}(Q)$ turns out to be noetherian.

Recall that an abelian category $\mathcal{G}$ is connected if it cannot be non-trivially written as a direct sum $\mathcal{G}_{1} \oplus \mathcal{G}_{2}$. The examples (E1)-(E5) are all connected and, remarkably, they are the only ones:

Theorem 7.2.1. [57] Let $\mathcal{C}$ be a connected noetherian Ext-finite hereditary category satisfying Serre duality over $k$. Then $\mathcal{C}$ is one of the categories (E1)-(E5).

Space does not permit us to sketch the fairly involved proof of this theorem. Suffice it to say that, after a series of reductions which in particular eliminate cases (E2)-(E5), one arrives at the situation where Theorem 2.1.5 can be invoked to show that $\mathcal{C}=\operatorname{qgr}(R)$ for some graded domain $R$ of quadratic growth. Then, amongst other facts, Theorem 5.1 is used to show that $\mathcal{C}$ is of the form (E1).

All the hypotheses for Theorem 7.2 .1 are necessary. For example, the noncommutative curves considered in [74] (see 97.3 below) satisfy $(\mathrm{C} 1)-(\mathrm{C} 3)$ but do not satisfy Serre duality. Nevertheless, it is tempting to ask whether a result similar to Theorem 7.2.1 remains valid without the noetherian or perhaps the Serre duality hypotheses. This may require working up to derived equivalence.

It is probably too early to be sure what are the "right" hypotheses to demand of a noncommutative analogue of a smooth proper variety. However, one indication that (C1)-(C4) may need to be supplemented is that, for example, they do not distinguish between algebraic and analytic (smooth compact) surfaces 1 A way around this problem is to use another subtle property of smooth proper schemes that was also discovered by Bondal and Kapranov and is not satisfied by analytic surfaces.

\footnotetext{
${ }^{1}$ The reason for the restriction to surfaces is that we do not know if for analytic varieties the Yoneda Ext-groups in $\operatorname{coh}(X)$ coincide with the traditional Ext-groups in the category of all $\mathcal{O}_{X^{-}}$ modules. Therefore we do not know if Serre duality in the sense of 7.1 holds for $\operatorname{coh}(X)$. For smooth compact analytic surfaces the equality of these Ext-groups can be proved directly [20].
} 
Recall that a cohomological functor $H: D^{b}(\mathcal{C}) \rightarrow \bmod (k)$ is of finite type if, for $A \in D^{b}(\mathcal{C})$, only a finite number of the $H(A[n])$ are non-zero. The appropriate condition is:

(C5) Let $\mathcal{C}$ be an Ext-finite abelian category of finite homological dimension. Then $\mathcal{C}$ is saturated if every cohomological functor $H: D^{b}(\mathcal{C}) \rightarrow \bmod (k)$ of finite type is of the form $\operatorname{Hom}(A,-)$ (i.e. $H$ is representable).

It was shown in [17] that $\operatorname{coh}(X)$ is saturated when $X$ is a smooth projective scheme and that saturation also holds for categories of the form $\bmod (\Lambda)$ with $\Lambda$ a finite dimensional algebra. It is easy to show that a saturated category satisfies Serre duality.

Combined with Theorem 7.2.1, this gives a much more compact classification.

Corollary 7.2.2. Assume that $\mathcal{C}$ is a saturated connected noetherian Ext-finite hereditary category. Then $\mathcal{C}$ has one of the following forms:

(1) $\bmod (\Lambda)$ where $\Lambda$ is an indecomposable finite dimensional hereditary algebra (this is very special case of (E5)).

(2) $\operatorname{coh}(\mathcal{O})$ where $\mathcal{O}$ is a sheaf of hereditary $\mathcal{O}_{X}$ orders (see (E1) above) over a smooth connected projective curve $X$.

It is easy to show that the abelian categories occurring in this corollary are of the form $\operatorname{qgr}(R)$ for $R$ a graded ring of Gelfand-Kirillov dimension $\leq 2$, and so this result can be regarded as a partial converse to the results of the last two sections. In a sense, we have therefore generalized the classical commutative result that smooth proper curves are projective.

Let us briefly turn to more general graded algebras. Although the saturation condition seems quite subtle, it is closely connected to the $\chi_{n}$ conditions we discussed at the end of $\$ 2.1$. The following result connects the saturation condition with graded rings. In this result we define a connected graded ring $R$ to have finite right cohomological dimension if the functor $\tau M=\lim _{\operatorname{Hom}}\left(R / R_{\geq n}, M\right)$ has finite cohomological dimension. If $(\mathcal{C}, \mathcal{O})=(\operatorname{qgr}(R), \pi R)$, this is the same as demanding that the functor $\Gamma(-)=\operatorname{Hom}(\mathcal{O},-)$ has finite cohomological dimension.

Theorem 7.2.3. [20] Let $R$ be a connected graded noetherian ring satisfying the following hypotheses:

1. $R$ and its opposite ring $R^{\circ}$ satisfy $\chi_{n}$ for all $n$;

2. $R$ and $R^{\circ}$ have finite right cohomological dimension;

3. $\operatorname{qgr}(R)$ has finite homological dimension.

Then $\operatorname{qgr}(R)$ is saturated.

We will discuss the first two hypotheses in more detail at the end of 77.4 . The subtlety of the saturation condition is indicated by the following example.

Proposition 7.2.4. [20] Assume that $X$ is an analytic $K 3$ surface with no curves. Then $\operatorname{coh}(X)$ is not saturated.

7.3. More curves. Yet another possible approach to noncommutative curves has been given by Smith and Zhang in [74.

If $X$ is a variety over $k$, then the (closed) points of $X$ are in one-one correspondence with the simple objects in $\mathrm{Qch}(X)$. Similarly if $Y \subset X$ is an irreducible curve, then its structure sheaf $\mathcal{O}_{Y}$ is a 1-critical object in $\mathrm{Qch}(X)$, that is, an object which is not of finite length but for which every quotient object has finite length (a 
finite length object is one that is a finite extension of simples). Now suppose that $\mathcal{C}$ is a locally noetherian Grothendieck category. If we want to view $\mathcal{C}$ as a noncommutative variety, then, by analogy, we may argue that the points of $\mathcal{C}$ should be the simple objects in $\mathcal{C}$ and the irreducible curves in $\mathcal{C}$ should be given by the 1 -critical objects. Two 1-critical objects should correspond to the same curve if they have a common non-trivial subobject.

Let $L$ be an irreducible curve in $\mathcal{C}$. To be consistent with our general philosophy we should associate an abelian category $\operatorname{Mod}(L)$ to $L$. Following [74, $\operatorname{Mod}(L)$ is defined to be the smallest abelian subcategory, containing $L$, of $\mathcal{C}$ which satisfies the following conditions.

- $\operatorname{Mod}(L)$ is closed under subquotients and direct sums.

- $\operatorname{Mod}(L)$ is closed under essential extensions by finite length objects.

In 74 it is shown that, if $(\mathcal{C}, L)$ satisfy suitable hypotheses which are special but which nevertheless hold in many examples, then $\operatorname{Mod}(L)$ is isomorphic to the quotient category $\mathcal{G}$ of $\mathbb{Z}^{t}$-graded $k\left[x_{1}, \ldots, x_{t}\right]$-modules, modulo those of Krull dimension $\leq(t-2)$. Categories like $\mathcal{G}$ have properties which are very close to those of $\mathbb{P}^{1}$ but they do not satisfy Serre duality when $t \neq 2$. This shows that the Serre duality condition may not always be satisfied, even in natural situations.

7.4. Serre duality. It is an unfortunate fact that the Serre duality condition (C4) from $\$ 7.1$ only makes sense for categories which have finite homological dimension, whereas in classical algebraic geometry much of the significance of Serre duality comes in applications to singular varieties. We expect the same to be true for noncommutative geometry and so in this section we describe a second notion of Serre duality that works more generally. This will naturally lead us into a discussion of various homological conditions, notably Gorenstein conditions, that will be important later.

Suppose that $\mathcal{C}$ is an Ext-finite noetherian abelian category with a distinguished object $\mathcal{O} \in \mathcal{C}$. By analogy with the commutative case we write $\Gamma(-)$ for $\operatorname{Hom}(\mathcal{O},-)$. Then $(\mathcal{C}, \mathcal{O})$ is defined to satisfy classical Serre duality if there exists an object $\omega^{\circ} \in D^{b}(\mathcal{C})$ together with a natural isomorphism

$$
\operatorname{RHom}\left(-, \omega^{\circ}\right) \cong \mathrm{R} \Gamma(-)^{*}
$$

where $*$ refers to the $k$-dual. Note that these derived functors can be computed using the closure $\widetilde{\mathcal{C}}$, as defined in $\$ 1.1$. Since $\omega^{\circ}$ represents a functor it is clear that it must be unique if it exists, and, moreover, its existence forces $\Gamma(-)$ to have finite cohomological dimension. Given that Serre duality is such a powerful tool in the commutative case, it would be interesting to know how much more generally it holds. The next results show that $\omega^{\circ}$ does exist for many noncommutative rings.

Theorem 7.4.1. 95] Let $(\mathcal{C}, \mathcal{O})=(\operatorname{qgr}(R), \pi R)$ for a noetherian connected graded ring $R$. Assume that $R$ satisfies:

(a) $R$ and its opposite ring $R^{\circ}$ satisfy $\chi_{n}$ for all $n$;

(b) $R$ and $R^{o p}$ have finite right cohomological dimension.

Then $(\operatorname{qgr}(R), \pi R)$ satisfies classical Serre duality.

It should be noted that, by invoking the Brown representability theorem [35], [51], Jorgensen has shown [28] that if we allow $\omega^{\circ}$ to lie in $D(\operatorname{QGr}(R))$, then it exists under far weaker hypotheses. Notably, his result does not require the $\chi$ assumption. 
Jorgensen, Yekutieli, Zhang and others have shown that, under the hypotheses of Theorem 7.4.1 a surprising number of results from the homological theory of commutative varieties do generalize to the noncommutative universe. See, for example, [29], [30], [31], [77], [94], [96] and the references therein.

It is not known how severe are the assumptions of Theorems 7.4.1 and 7.2.3 In particular, it is not known whether every noetherian connected graded ring $R$ has finite cohomological dimension, as would be true if $R$ were commutative [26] Theorem III.2.7]. In contrast, Theorem 5.6 produces many rings $R$ that do not satisfy the $\chi$ conditions. One can easily circumvent those examples by demanding that the noetherian prime ring $R$ is a maximal order, in the sense that there exists no ring $R \subsetneq R^{\prime} \subset Q(R)$ with $a R^{\prime} b \subseteq R$, for some units $a, b \in Q(R)$. (This is the appropriate noncommutative analogue of an integrally closed commutative noetherian ring.) Notice that the way we constructed the example $S=k+U y$ in (2.3) ensures that it cannot be a maximal order, and the same is true of the rings created by Theorem 5.6. It remains an open question whether every noetherian connected graded maximal order satisfies $\chi$.

Here is one case where the answer is known. A ring $R$ is called Artin-Schelter $(A S)$ Gorenstein if $R$ has finite left and right injective dimension $d$ and, for some shift $m$, one has

$$
\operatorname{Ext}^{i}(k, R)= \begin{cases}0 & \text { if } i \neq d \\ k(m) & \text { if } i=d .\end{cases}
$$

Then [95] shows that noetherian AS Gorenstein rings of dimension $d+1$ satisfy the hypotheses of Theorem 7.4.1. Moreover, $\omega^{\circ}$ is of the form $\omega[d]$ where $\omega$ is the image of an honest $R$-bimodule that is left and right free of rank one. It follows that, in the case of AS-Gorenstein rings, Serre duality takes the form of natural isomorphisms

$$
\operatorname{Ext}_{\mathrm{qgr} R}^{i}(-, \omega) \cong R^{d-i} \Gamma(-)^{*} .
$$

This is essentially the classical statement of Serre duality for projective varieties [26, Theorem III.7.6].

\section{Artin-Schelter Regular algebras}

The area of mathematics described in this survey really began some fifteen years ago in the work [3] of Artin and Schelter, where they attempted to classify the appropriate analogues of a commutative polynomial ring in three variables. The final solution to this problem in [6], 7] for rings generated in degree one and in [79], 80] for the general case required the creation of many of the ideas described in the earlier sections of this paper and so it serves as a convenient bridge between our expositions on noncommutative curves and on noncommutative surfaces.

The philosophy of Artin and Schelter is that a noncommutative projective plane should be represented by a ring with some of the most basic properties of a polynomial ring in three variables. This point of view is essentially ad hoc but an a posteriori justification, which will be discussed in $\$ 11$ has been provided by the work of Bondal and Polishchuk in [19]. In the approach of [3], it is more natural to allow the generators of that polynomial ring to have varying weights, but this can be regarded as an advantage, since we will obtain (some) noncommutative quadrics and more general weighted projective spaces by the same technique! 
So what should be the definition of our noncommutative analogue of a polynomial ring in three variables? Obviously, it should be a connected graded ring of homological dimension three and have finite Gelfand-Kirillov dimension. By themselves, these conditions are insufficient, since they do not exclude some particularly unpleasant rings like $k\{x, y\} /(x y)$. The solution is to introduce the Gorenstein condition mentioned in the last section. More precisely:

Definition 8.1. A connected graded ring $A$ is called Artin-Schelter (AS) regular of dimension $d$ if it satisfies the following conditions:

(1) $A$ has homological dimension $d$.

(2) $\operatorname{GKdim}(A)<\infty$.

(3) $A$ is AS Gorenstein.

The advantage of the third hypothesis, for AS-regular rings of dimension 3, is that the projective resolution of $k$ is forced to be of the form

$$
0 \longrightarrow A \longrightarrow A^{n} \longrightarrow A^{n} \longrightarrow A \longrightarrow k \longrightarrow 0
$$

(where the shifts in degree have been ignored) for some $n$, and this quickly gives one strong information on the Hilbert series of $A$. Using this, Artin, Tate, Van den Bergh and Stephenson gave a complete classification for AS regular rings of dimension three.

Theorem 8.2. ([6], 7], [79], 80]) The AS-regular rings A of dimension three can be classified. They are all noetherian domains with the Hilbert series of a weighted polynomial ring $k[x, y, z]$ - thus the $(x, y, z)$ can be given degrees $(a, b, c)$ other than $(1,1,1)$.

One of the more pleasant aspects of this classification is that the noetherian condition comes for free once one makes the Gorenstein hypothesis. Conversely, it could be that the Gorenstein condition is automatic for noetherian rings of finite homological dimension [81.

In order to give an idea of the classification in Theorem 8.2 consider the case when the variables all have weight 1 , so $A$ has Hilbert series $(1-t)^{-3}$. (The proof in the general case is similar.) Here, once again, one looks at $\operatorname{qgr}(A)$. The idea is to look at the simplest possible objects in $\operatorname{qgr}(A)$, the (images of) point modules, modules $M=\bigoplus_{i>0} M_{i}$ that are generated by $M_{0}$ and satisfy $\operatorname{dim}_{k} M_{i}=1$, for all $i \geq 0$. If $A$ were commutative, then such a module would be isomorphic to a polynomial ring in one variable and hence be isomorphic to the homogeneous coordinate ring of a point $p \in \operatorname{Proj}(A)$. Hence the name. When $A$ is noncommutative it is easy to understand the point modules combinatorially and this leads to the main step in the proof of Theorem 8.2 either $A \cong B\left(\mathbb{P}^{2}, \mathcal{L}, \sigma\right)$ for some $\mathcal{L}$ and $\sigma$ or there exists a homomorphic image $B$ of $A$ isomorphic to $B(E, \mathcal{L}, \sigma)$ for some (possibly singular or non-reduced) elliptic curve $E$. Moreover, $B$ is defined by 3 quadratic relations together with one cubic $\tilde{g}$ and so $A$ is defined by those 3 quadratic relations. Notably, $A$ is uniquely determined by $(E, \mathcal{L}, \sigma)$ ! Finally, one can show that image $g$ of $\tilde{g}$ in $A$ is a non-zero divisor that is normal in the sense that it satisfies $g A=A g$. Thus, $B=A / g A$ and this enables one to use one's detailed knowledge of the structure of $B$ to prove the required properties for $A$.

It is clearest if we explain this procedure for an explicit example and we use

$$
A=k\left\{x_{1}, x_{2}, x_{3}\right\} /\left(x_{2} x_{1}-\alpha x_{1} x_{2}, x_{3} x_{1}-\beta x_{1} x_{3}, x_{3} x_{2}-\gamma x_{2} x_{3}\right),
$$


where $\alpha, \beta, \gamma \in k^{*}$. The reader may recognize this ring as a typical example of a "quantum coordinate ring of affine 3 -space". Let $M=\bigoplus_{i \geq 0} m_{i} k$ be a point module for $A$, with module structure defined by $m_{i} x_{j}=\lambda_{i j} m_{i+1}$ for $\lambda_{i j} \in k$. Each relation between the generators of $A$ must kill $m_{0}$ (or any $m_{i}$ ) and this leads to the following three equations in the $\lambda_{i j}$ :

$$
\lambda_{02} \lambda_{11}-\alpha \lambda_{01} \lambda_{12}, \lambda_{03} \lambda_{11}-\beta \lambda_{01} \lambda_{13}, \lambda_{03} \lambda_{12}-\gamma \lambda_{02} \lambda_{13} .
$$

These equations may be rewritten as $N \cdot \lambda=0$, where (ignoring the first subscript)

$$
N=N(\lambda)=\left(\begin{array}{ccc}
\lambda_{2} & -\alpha \lambda_{1} & 0 \\
\lambda_{3} & 0 & -\beta \lambda_{1} \\
0 & \lambda_{3} & -\gamma \lambda_{2}
\end{array}\right) \text { and } \lambda=\left(\begin{array}{c}
\lambda_{1} \\
\lambda_{2} \\
\lambda_{3}
\end{array}\right)
$$

This defines a subvariety $\Lambda \subseteq \mathbb{P}^{2} \times \mathbb{P}^{2} \equiv \mathbb{P}\left(A_{1}^{*}\right) \times \mathbb{P}\left(A_{1}^{*}\right)$. A little linear algebra shows that $\Lambda$ is the graph of an automorphism $\sigma$ of a subvariety $E \subseteq \mathbb{P}^{2}$. Indeed,

$$
E=\{\lambda \mid \operatorname{det}(N(\lambda))=0\}=\left\{\left(\lambda_{1}, \lambda_{2}, \lambda_{3}\right) \mid(\beta-\alpha \gamma) \lambda_{1} \lambda_{2} \lambda_{3}=0\right\} .
$$

Similarly, $\sigma$ is defined by $\sigma(\lambda)=\{\mu: N(\lambda) \cdot \mu=0\}$. We leave it to the reader to check that $\mu$ is uniquely determined and to determine the precise form of $\sigma$. Since $E$ is determined by the point modules of $A$ it is often called the point scheme $\mathcal{P}(A)$ of $A$.

Now form the twisted homogeneous coordinate ring $B=B(E, \mathcal{O}(1), \sigma)$. Then, $B_{1}=\mathrm{H}^{0}(\mathcal{O}(1))$ is naturally isomorphic to $A_{1}$ and it is an easy exercise to show that this induces a homomorphism $\phi: A \rightarrow B$. The details can be found in [7] or [72. Lemma 3.3] but the idea is as follows: Given a quadratic relation $f=\sum \alpha_{i j} x_{i} x_{j}$ we need to prove that $\phi(f)=0$. By the definition of multiplication in $B$ this amounts to proving that, if $\left\{\bar{x}_{i}: 1 \leq i \leq 3\right\}$ denote the coordinate functions on $\mathbb{P}^{2}$, then

$$
\sum \alpha_{i j} \bar{x}_{i}(p) \bar{x}_{j}\left(p^{\sigma}\right)=0 \quad \text { for all } p \in E .
$$

Now, $\Lambda$ was defined as the common zeros of the linearizations $\tilde{f}=\sum \alpha_{i j} x_{0 i} x_{1 j}$ of all such relations $f$. Thus, $\tilde{f}$ is zero on $\Lambda$ which, since $\Lambda=\left\{\left(p, p^{\sigma}\right): p \in E\right\}$, implies that $\tilde{f}$ is zero on $\left\{\left(p, p^{\sigma}\right): p \in E\right\}$. This is equivalent to (8.3). Finally, an application of the Riemann-Roch theorem can then be used to prove that $B$ is generated by $B_{1}$ and hence that $\phi$ is surjective. Thus, $B$ is a homomorphic image of $A$. The remarkable thing about Theorem 8.2 is that this simple approach actually works for all AS regular algebras of dimension three.

Note that we really have two different types of rings in (8.2). If $\beta=\alpha \gamma$, then $E \cong \mathbb{P}^{2}$ and indeed $A \cong B$. Thus, $\operatorname{qgr}(A) \cong \mathbb{P}^{2}$, by Theorem 3.2. If $\beta \neq \alpha \gamma$, then $E=\left\{\lambda_{1} \lambda_{2} \lambda_{3}=0\right\}$ is the product of three lines and one can check that $B=A / x_{1} x_{2} x_{3} A$. Thus Theorem 3.2 now says that $\operatorname{coh}(E) \cong \operatorname{qgr}(B)$ embeds into $\operatorname{qgr}(A)$. A natural interpretation is to regard $E$ as embedded in the "noncommutative projective plane" $\operatorname{qgr}(A)$. One can extend this analogy by observing that the (suitably defined) complement $\operatorname{qgr}(A) \backslash E \cong \bmod (R)$, where $R=A\left[g^{-1}\right]_{0}$ and so $\bmod (R)$ is a "noncommutative affine space". We reiterate that this phenomenon is typical of AS regular algebras of dimension three and illustrates the assertion from the introduction that all known noncommutative projective surfaces contain an embedded commutative curve. We will return to this point of view in $\$ 11.2$

The most interesting examples of AS regular algebras of dimension three are the so-called 3-dimensional Sklyanin algebras. 
Example 8.3. The "generic" case, when $E$ is a smooth elliptic curve and $\sigma$ is given by translation under the group law, corresponds to the Sklyanin algebra

$$
\operatorname{Skl}_{3}(a, b, c)=k\left\{x_{0}, x_{1}, x_{2}\right\} /\left(a x_{i} x_{i+1}+b x_{i+1} x_{i}+c x_{i+2}^{2}: i=1,2,3 \bmod 3\right),
$$

where $(a, b, c) \in \mathbb{P}^{2} \backslash F$, for a (known) finite set $F$.

The reader may like to repeat the earlier analysis and show that the associated curve for $\mathrm{Skl}_{3}=\mathrm{Skl}_{3}(a b c)$ is the smooth elliptic curve

$$
E: \quad a b c\left(x_{1}^{3}+x_{2}^{3}+x_{3}^{3}\right)-\left(a^{3}+b^{3}+c^{3}\right) x_{1} x_{2} x_{3}=0 .
$$

Thus, $E$ embeds into $\operatorname{qgr}\left(\mathrm{Skl}_{3}\right)$. An interesting challenge is to find a direct algebraic proof that $\mathrm{Skl}_{3}$ has Hilbert series $(1-t)^{-3}$. The only known proofs use the geometric techniques of this survey.

The results on three dimensional AS-regular rings have been extended to a certain extent to higher dimension. Space does not permit us to discuss this in any detail so we will suffice by saying that, well before Artin-Schelter, Sklyanin had constructed some putative four-dimensional algebras (see [67, 68] and Example 8.5. below) which were then generalized by Odesskii and Feigin [52], [53]. These are now called higher dimensional Sklyanin algebras. That these algebras have the expected properties was shown in a series of papers [44], 72], 73], [82. However, in contrast to Theorem 8.2 there seem to exist a great many other four-dimensional AS-regular rings, and so there is no classification in sight.

It is customary to divide the class of four-dimensional AS-regular algebras $R$ (generated in degree one) into subclasses according to the point scheme $\mathcal{P}(R) \subseteq \mathbb{P}^{3}$ parametrizing the point modules. The case where $\mathcal{P}(R)$ contains a quadric is best understood [66, 64]. Many examples [43], 67], 75], including the 4 dimensional Sklyanin algebra, have been constructed where $\mathcal{P}(R)$ is the union of an elliptic curve and a finite set of points. However, it is also possible for $\mathcal{P}(R)$ to consist of just a finite set -indeed this is presumably the generic case. Very little is known about the general properties of this class. For example, all known rings $R$ with a finite point scheme are finitely generated as modules over their center [85], but it seems hard to believe that this could be true in general.

Remark 8.4. Bondal has proved (unpublished) that any Poisson bracket on $\mathbb{P}^{3}$ has at least a one-dimensional zero-locus. Thus the existence of four dimensional ASregular rings with a finite number of points indicates that intuition obtained from the quasi-classical case is not always reliable.

In the later sections we will use factor rings of the 4-dimensional Sklyanin algebra as examples of quadric surfaces and so we end this section by collecting together the facts we need. For more details about this algebra and its rich module structure, we refer the reader to [44], [72], [73] or the survey [71].

Example 8.5. The 4-dimensional Sklyanin algebra $\mathrm{Skl}_{4}$ is the $k$-algebra with 4 generators $x_{0}, \ldots, x_{3}$ and the 6 relations

$$
x_{0} x_{i}-x_{i} x_{0}=\alpha_{i}\left(x_{i+1} x_{i+2}+x_{i+2} x_{i+1}\right), \quad x_{0} x_{i}+x_{i} x_{0}=x_{i+1} x_{i+2}-x_{i+2} x_{i+1},
$$

where the subscripts are taken to be $\{1,2,3\} \bmod 3$ and the $\alpha_{i}$ satisfy $\alpha_{1} \alpha_{2} \alpha_{3}+$ $\alpha_{1}+\alpha_{2}+\alpha_{3}=0$ and $\left\{\alpha_{i}\right\} \cap\{0, \pm 1\}=\emptyset$. The significant properties are that $S=\mathrm{Skl}_{4}$ is an AS regular algebra of dimension 4 that has a two-dimensional space $\Omega$ of central homogeneous elements in $S_{2}$. The factor ring $S /(\Omega)$ is isomorphic to a 
twisted homogeneous coordinate $\operatorname{ring} B(E, \mathcal{L}, \sigma)$, where $E$ is a smooth elliptic curve, $\sigma$ is an automorphism given by translation, but $\mathcal{L}$ is now a line bundle of degree 4. Since this data actually determines the Sklyanin algebra, we sometimes write $\mathrm{Skl}_{4}=\operatorname{Skl}_{4}(E, \mathcal{L}, \sigma)$. In fact, $\operatorname{Skl}_{4}(E, \mathcal{L}, \sigma)$ is even determined up to isomorphism by $(E, \sigma)$, since $\mathcal{L}$ just describes the presentation of the algebra as a factor of a free algebra.

Each $\Omega_{\lambda} \in \mathbb{P}(\Omega)$ generates a prime ideal of $\mathrm{Skl}_{4}$ and we write $R_{\lambda}=R_{\lambda}(E, \mathcal{L}, \sigma)$ for the factor $\mathrm{Skl}_{4} / \Omega_{\lambda} \mathrm{Skl}_{4}$.

\section{Commutative surfaces}

9.1. Overview. After the classification of curves, the next classical subject in algebraic geometry is the classification of surfaces. Unfortunately this is a much more difficult subject and the information is far less complete. In this section we briefly survey this theory while in the remainder of the paper we will discuss the extent to which those results generalize to the noncommutative universe.

For the rest of the paper, a "surface" will be a smooth proper connected scheme of dimension two over $k$. It is well-known that such a surface is automatically projective.

Since the classification of surfaces $X$ is too hard to tackle directly, one first classifies them up to birational equivalence - this just means that one classifies the function fields $k(X)$. Of course, $k(X)$ is just an extension of $k$ of transcendence degree two. If $k(X) \cong k(x, y)$, we call $X$ a rational surface. A convenient interpretation of birational equivalence is given by the theory of minimal models: if $X$ is a surface, then $X$ is minimal if every surjective map $X \rightarrow Y$ to a birational surface $Y$ is an isomorphism. Every surface is birationally equivalent to a minimal one and this minimal model is unique except for ruled and rational surfaces. For rational surfaces the minimal models are respectively $\mathbb{P}^{2}$ and $\mathbb{P}^{1}$-bundles over $\mathbb{P}^{1}$ (these are also known as Hirzebruch surfaces) [14 Thm V.10]. For more details, we refer the reader to [13, [14, 26].

By contrast, the classification of surfaces within a birational equivalence class has a much neater answer. A classical result by Zariski states that if $X$ and $X^{\prime}$ are surfaces which are birationally equivalent, then it is possible to go from $X$ to $X^{\prime}$ by repeatedly applying blowing-ups and blowing-downs.

As we will want to mimic the procedure later, we first describe blowing up/down in more detail (see, also, [26, $\S \mathrm{V} .3]$ ). Let $p \in X$ be a point on the surface $X$, with corresponding maximal ideal $\mathfrak{m}$. Then the blow-up $\widetilde{X}$ of $x$ in $X$ is defined as Proj $\mathcal{D}$ where

$$
\mathcal{D}=\mathcal{O}_{X} \oplus \mathfrak{m} \oplus \mathfrak{m}^{2} \oplus \cdots,
$$

thought of as a sheaf of $\mathcal{O}_{X}$-algebras on $X$ (for the construction of Proj of a sheaf of graded algebras see [26]). Since blowing up is an essentially local transformation, it is enough to analyse this in the case when $X=\operatorname{Spec} k[x, y]$ and $p$ is the origin. After some elementary computations one obtains

$$
\tilde{X}=\operatorname{Spec} k[y / x, x] \cup \operatorname{Spec} k[x / y, y] .
$$

It follows that, away from $p$, the map $\tilde{X} \rightarrow X$ is an isomorphism. However the inverse image $L$ of $p$ is isomorphic to $\mathbb{P}^{1}$ and has self intersection -1 [26. Proposition V.3.1]. The curve $L$ is called the exceptional curve. More generally, if $Y$ is an 
arbitrary surface, then a curve $L \subset Y$ is called exceptional if $L \cong \mathbb{P}^{1}$ and if $L$ has self intersection -1 .

The inverse to blowing up is blowing down for which, given an exceptional curve $L$ on a surface $Y$, one constructs a map (determined up to a unique isomorphism) $\alpha: Y \rightarrow X$ to some surface $X$ such that $\alpha$ contracts $L$ to a point and is an isomorphism outside $L$. One way of constructing $\alpha$ is outlined below, and this technique also provides some birational transformations of a more global nature than just blowing up or down.

Thus, let $\mathcal{L}$ be a line bundle on a surface $X$ and fix nonzero global sections $x_{0}, \ldots, x_{n} \in \mathrm{H}^{0}(X, \mathcal{L})$. If $\mathcal{L}$ is generated by the $x_{i}$, then they define a map $x: X \rightarrow$ $\mathbb{P}^{n}$ such that $\mathcal{L}=x^{*} \mathcal{O}(1)$ and such that the $x_{i}$ are the pullbacks of the coordinate functions 26 Theorem II.7.1]. Let $X^{\prime}$ denote the image of $X$. In general there is not much to be said about $X^{\prime}$ although, in some very nice cases, $x$ will just contract a finite number of exceptional curves. In particular, if $L$ is an exceptional curve on $X$, then one can pick $\mathcal{L}$ such that $x$ contracts precisely $L$ and nothing else [26. proof of Theorem V.5.7]. This is the classical construction of blowing down.

Suppose now that $\mathcal{L}$ is not generated by $x_{0}, \ldots, x_{n}$ and let $\mathcal{F}$ be the subsheaf of $\mathcal{L}$ generated by those sections. In this case, it is convenient to replace $\mathcal{L}$ by $\mathcal{F}^{* *}$ so that we may assume that $\mathcal{L} / \mathcal{F}$ has finite length. In other words, if

$$
U=\left\{p \in X \mid \mathcal{L} \text { is generated by } x_{0}, \ldots, x_{n} \text { in } p\right\},
$$

then $X \backslash U$ is a finite set of points and $x$ defines a map of algebraic varieties $x: U \rightarrow \mathbb{P}^{n}$. Classically, one calls this a rational map $x: X \rightarrow \mathbb{P}^{n}$. We define the image $X^{\prime}$ of $x$ to be the Zariski-closure of $x(U)$ in $\mathbb{P}^{n}$.

In the following three subsections we discuss some classical birational transformations for which we will be able to construct noncommutative analogues in $\$ 13$.

9.2. Cubic surfaces [26, Proposition 4.8]. Let $X=\mathbb{P}^{2}$ and let $p_{1}, \ldots, p_{6} \in \mathbb{P}^{2}$ be six points in general position. By this we mean that no two points are equal, no three lie on a line and not all six lie on a conic. Then the space of sections of $\mathcal{O}(3)$ (cubic forms) vanishing in $p_{1}, \ldots, p_{6}$ is four dimensional and these sections generate $\mathcal{O}(3)$ on $\mathbb{P}^{2} \backslash\left\{p_{1}, \ldots, p_{6}\right\}$. As explained above we obtain a corresponding rational map $x: \mathbb{P}^{2} \rightarrow \mathbb{P}^{3}$. In this case it turns out that the image of $x$ is a cubic surface in $\mathbb{P}^{3}$ and it is obtained from $\mathbb{P}^{2}$ by blowing up the six points $p_{1}, \ldots, p_{6}$. It is a classical fact $[45, \S 24]$ that any cubic surface in $\mathbb{P}^{3}$ can be obtained in this way.

9.3. The Cremona transformation [26, Example V.4.2.3]. Fix three points $p, q, r \in \mathbb{P}^{2}$ that are not collinear. The space of sections of $\mathcal{O}(2)$ (quadratic forms) vanishing in those three points is three dimensional and so we now obtain a rational map $x: \mathbb{P}^{2} \rightarrow \mathbb{P}^{2}$. In this case $x$ is birational and is classically called a Cremona transformation.

If we choose homogeneous coordinates $x, y, z$ and we put $p, q, r$ in the standard positions $(1,0,0),(0,1,0),(0,0,1)$, then this Cremona transformation is given by $(x, y, z) \mapsto(y z, z x, x y)$. From this explicit description it follows easily that the Cremona transformation is the composition of the following birational transformations.

(a) First blow up the points $p, q, r$ to obtain a surface $\widetilde{X}$.

(b) It follows from general theory that the three lines connecting the points $p, q, r$ become exceptional and disjoint in $\widetilde{X}$. Now blow down these exceptional lines. 
9.4. Quadrics [26, Exercise V.4.1]. Now we choose two distinct points $p, q \in \mathbb{P}^{2}$ and we look at the four dimensional space of sections of $\mathcal{O}(2)$ which vanish in $p, q$. This gives us a rational map $x: \mathbb{P}^{2} \rightarrow \mathbb{P}^{3}$ whose image is a quadric surface. In this case $x$ is obtained from blowing up $p, q$ and then blowing down the connecting line.

The inverse to $x$ (as a birational map) can be constructed as follows. Every quadric is isomorphic to $\mathbb{P}^{1} \times \mathbb{P}^{1}$. Fix some point $r \in \mathbb{P}^{1} \times \mathbb{P}^{1}$ and consider the three dimensional space of sections of $\mathcal{O}(1,1)$ vanishing in $r$. This gives us a rational map $y: \mathbb{P}^{1} \times \mathbb{P}^{1} \rightarrow \mathbb{P}^{2}$ which is the inverse to $x$.

9.5. Some reformulations. Unfortunately the constructions exhibited in 99.1 do not generalize well to the noncommutative case and so we will give a more suitable reformulation.

Let $X$ be a surface and let $\mathcal{L}$ be a line bundle on $X$ generated by global sections. Then $\mathcal{L}$ defines a map $x: X \rightarrow \mathbb{P}^{n}$ and in 9.1 we have considered $X^{\prime}=x(X)$. With an eye towards noncommutative generalizations, it is better to consider $\widetilde{X}=\operatorname{Proj} A$, where $A$ is the ring $\bigoplus_{n} H^{0}\left(X, \mathcal{L}^{\otimes n}\right)$. To understand the relation between $X^{\prime}$ and $\tilde{X}$ we introduce the Stein factorization $x: X \stackrel{\alpha}{\rightarrow} Y \stackrel{\beta}{\rightarrow} \mathbb{P}^{n}$ of $x$. This factorization has the defining property that $\beta$ is finite and $\alpha_{*}\left(\mathcal{O}_{X}\right)=\mathcal{O}_{Y}$. Put $\mathcal{L}_{Y}=\beta^{*} \mathcal{O}(1)$. This yields

$$
\begin{aligned}
H^{0}\left(X, \mathcal{L}^{\otimes n}\right) & =H^{0}\left(X, \alpha^{*}\left(\mathcal{L}_{Y}\right)^{\otimes n}\right)=H^{0}\left(Y, \alpha_{*} \alpha^{*}\left(\mathcal{L}_{Y}\right)^{\otimes n}\right) \\
& =H^{0}\left(Y, \alpha_{*} \mathcal{O}_{X} \otimes \mathcal{O}_{Y} \mathcal{L}_{Y}^{\otimes n}\right)=H^{0}\left(Y, \mathcal{L}_{Y}^{\otimes n}\right) .
\end{aligned}
$$

Since $\beta$ is finite, $\mathcal{L}_{Y}$ is ample and so, by Serre's Theorem 2.1.2, we obtain $\widetilde{X}=Y$. Hence $\widetilde{X}$ is a finite cover of $X^{\prime}$.

If $\mathcal{L}$ is not generated by its global sections, then, as in 9.1 we let $\mathcal{F}$ be the subsheaf of $\mathcal{L}$ spanned by the global sections of $\mathcal{L}$ and we define $\widetilde{X}=\operatorname{Proj} A$ for $A=\bigoplus H^{0}\left(X, \mathcal{F}^{n}\right)$. Here $\mathcal{F}^{n}$ is, by definition, the image of $\mathcal{F}^{\otimes n}$ in $\mathcal{L}^{n}$. As in the previous paragraph, one can show that $\tilde{X}$ is a finite cover of $X^{\prime}$.

In the standard examples that were considered in $\S \S 9.29 .4, \tilde{X}=X^{\prime}$.

\section{Noncommutative SURFACES}

We start this section with the embarrassing admission that we do not really know what the correct definition should be for a noncommutative surface. Keeping with the philosophy of $\$ 95-17$ it would be tempting to accept one of the following definitions, the first of which is more algebraic while the second, inspired by [18], is more geometric.

Definition 10.1. (Attempt I) A noncommutative projective (normal but possibly singular) surface $\mathcal{C}$ is a category of the form $\operatorname{QGr}(A)$ where $A$ is a noetherian connected graded domain of Gelfand-Kirillov dimension three such that $A$ is a maximal order. Moreover, $\mathcal{C}$ is smooth if $\mathrm{QGr}(A)$ has homological dimension 2.

Definition 10.2. (Attempt II) A smooth noncommutative surface is a locally noetherian Grothendieck category of homological dimension two such that the full subcategory of noetherian objects is Ext-finite and saturated.

We should make some comments about Definition 10.1] Probably one should work with prime rings, but this introduces technical complications unrelated to the underlying intuition, as was true in 96 vis-a-vis 95. The assumption that $A$ is a maximal order is natural, given the discussion in \$7.4. Finally, this definition tacitly 
assumes the conjecture (see [4, p. 233]) that $2<\operatorname{GKdim}(A)<3$ is impossible for a connected graded domain $A$. By [4, Theorem 0.1] it is known that $2<\operatorname{GKdim}(A)<$ $2 \frac{1}{5}$ is impossible.

Unfortunately, at this point it seems to be completely intractable to obtain any substantial classification results starting from these definitions, so the precise definition is moot. Instead, the study on noncommutative surfaces has been more involved with an attempt to extend some basic parts of the commutative theory. This is what we will discuss in the forthcoming sections. In particular, as we will describe, there are good arguments that all noncommutative $\mathbb{P}^{2}$ 's and quadrics have been described and that, at least for important special cases, we have the right analogue of blowing up and down.

10.1. Birational classification. Mimicking the commutative theory we should first obtain a birational classification for noncommutative surfaces. However there are immediately some foundational questions here.

If we accept Definition 10.1 then the corresponding function field is $Q(A)_{0}$, in the notation of $\$ 5$. For Definition 10.2 the function field should presumably be defined as the endomorphism ring of the direct sum of the maximal injectives, but it is not clear that this notion is well behaved. Alternatively, we can concentrate initially on the classification of division algebras $D$ of transcendence degree two. Here, we use the definition of transcendence degree from [23] (essentially, this means that $D$ is the Goldie quotient ring of a finitely generated, (ungraded) domain $R$ of Gelfand-Kirillov dimension 2). Hopefully this is equal to the more sophisticated definition from [97], for which more favorable properties have been proved. For other types of transcendence degree of a more homological nature see [58, 63.

If we try to write down a list of known division algebras of transcendence degree two, then the list is surprisingly short:

(a) Division algebras which are finite over a central commutative subfield of transcendence degree two.

(b) Division rings of fractions of skew polynomial extensions of $k(X)$, for curves $X$ [21, p. 439].

(c) The function fields $Q\left(\mathrm{Skl}_{3}\right)_{0}$ of the three dimensional Sklyanin algebras $\mathrm{Skl}_{3}$.

Since no other examples are known, Mike Artin makes the provocative conjecture in 2 that these are in fact all the division algebras of transcendence degree two. The first author of this survey feels that this conjecture is false, whereas the second author, being more diplomatic, is neutral!

Anyway, true or not, this conjecture immediately gives rise to some interesting algebraic problems:

Example 10.1.1. Let $E$ be the function field of the "affine quantum plane". That is, $E$ is generated as a division algebra by two variables $u, v$ satisfying the relation $v u=q u v$ for some $q \in k^{*}$ which is not a root of unity. Let $\tau$ be the automorphism of $E$ given by $u \mapsto u^{-1}, v \mapsto v^{-1}$ and put $D=E^{\tau}$. Then $D$ is a division algebra of transcendence degree two and the question is where it fits in the above list. It turns out that $D$ is also the function field of a two-dimensional affine quantum plane and this was first proved using the techniques outlined in this survey. The details will be given in 13.6 . 


\section{Noncommutative PROJECTIVE PLANES AND QUADRICS}

Starting with this section we will take our first steps into the realm of noncommutative surfaces. In order to motivate the definition of the two most basic examples, namely the noncommutative analogues of $\mathbb{P}^{2}$ and $\mathbb{P}^{1} \times \mathbb{P}^{1}$, we discuss an extension of Theorem 2.1.5 This material was to some extent inspired by [19.

11.1. Extension of the Artin-Zhang Theorem. Theorem 2.1.5 depends on the availability of a suitable autoequivalence $s$ which sometimes makes it hard to apply. Inspection of the condition for ampleness of $s$ reveals that it only depends on the objects $\mathcal{O}(n)=s^{n} \mathcal{O}$ and not on $s$ itself. On the other hand, the construction of the ring $B$ depends on $s$. If we are willing to replace the notion of a $\mathbb{Z}$-graded $k$ algebra by the weaker notion of a $\mathbb{Z}$-algebra, then we can get rid of $s$ altogether. In general a $\mathbb{Z}$-algebra is just a $k$-linear category whose objects are indexed by $\mathbb{Z}$ and a module over a $\mathbb{Z}$-algebra is a $k$-linear functor to the category of $k$-vector spaces. The idea of regarding additive categories as generalizations of rings is standard. See for example [49].

Let us now spell out the abstract definition of a $\mathbb{Z}$-algebra in concrete terms. In the sequel a $\mathbb{Z}$-algebra [19] will be a $k$-algebra $A$ (usually without unit) together with a decomposition $A=\bigoplus_{i j \in \mathbb{Z}} A_{i j}$ such that the multiplication has the property that $A_{i j} A_{j k} \subset A_{i k}$ and $A_{i j} A_{k l}=0$ if $j \neq k$. It is clear that the $A_{i i}$ are subalgebras of $A$. We require that these have units $e_{i}$ such that if $a \in A_{i j}$, then $e_{i} a=a=a e_{j}$. Following [19] our point of view will be that $\mathbb{Z}$-algebras are generalizations of $\mathbb{Z}$ graded rings in the following sense: if $B=\bigoplus_{i \in \mathbb{Z}} B_{i}$ is a $\mathbb{Z}$-graded $k$-algebra, then putting $A_{i j}=B_{j-i}$ defines a $\mathbb{Z}$-algebra. Most properties of graded rings can be stated and proved in the more general context of $\mathbb{Z}$-algebras.

Given a $\mathbb{Z}$-algebra $A$, then a right $A$-module will be an ordinary right $A$-module $M$ together with a decomposition $M=\bigoplus M_{i}$ such that $M_{i} A_{i j} \subset M_{j}$, the $e_{i}$ act as units on $M_{i}$ and $M_{i} A_{j k}=0$ if $i \neq j$. We denote the category of right $A$ modules by $\operatorname{Gr}(A)$. It is clear that $\operatorname{Gr}(A)$ is a Grothendieck category and that, if $A$ is obtained from a $\mathbb{Z}$-graded ring $B$, then $\operatorname{Gr}(A) \simeq \operatorname{Gr}(B)$. We will say that $A$ is noetherian if $\operatorname{Gr}(A)$ is a locally noetherian category. We borrow other notions from the theory of graded rings. For example, $M \in \operatorname{Gr}(A)$ is called right bounded if $M_{i}=0$ for $i \gg 0$. We say that $M$ is torsion if it is a direct limit of right bounded objects. We denote the corresponding category by $\operatorname{Tors}(A)$ and set $\mathrm{QGr}(A)=\operatorname{Gr}(A) / \operatorname{Tors}(A)$.

Now consider a locally noetherian Grothendieck category $\mathcal{C}$ and let $(\mathcal{O}(n))_{n \in \mathbb{Z}}$ be a series of noetherian objects in $\mathcal{C}$. Associated to the $\mathcal{O}(n)$ there is a $\mathbb{Z}$-algebra $A$ given by $A_{i j}=\operatorname{Hom}(\mathcal{O}(-j), \mathcal{O}(-i))$ for $j \geq i, A_{i j}=0$ otherwise, and multiplication defined by composition. We say that $(\mathcal{O}(n))_{n}$ is ample if the conditions of Definition 2.1.4 hold.

We have the following obvious analogue of Theorem 2.1.5.

Theorem 11.1.1. Keep the above notation and assume that $\mathcal{C}$ has finite dimensional Hom's between noetherian objects. If $(\mathcal{O}(n))_{n}$ is ample, then $\mathcal{C} \simeq \operatorname{QGr}(A)$. In addition $A$ is noetherian.

11.2. Noncommutative planes. Following Artin and Schelter we propose the following definition of a noncommutative $\mathbb{P}^{2}$. It is somewhat more convenient to work with QGr rather than qgr but, by $\$ 1.1$, the difference is not significant. Although $\mathbb{Z}$-algebras do not appear in its definition, they will appear in its justification. 
Definition 11.2.1. A noncommutative $\mathbb{P}^{2}$ is a Grothendieck category of the form $\mathrm{QGr}(A)$, where $A$ is an AS regular algebra of dimension three, with Hilbert series $(1-t)^{-3}$.

Let $A$ be such an AS regular algebra. From the discussion in $\$ \mathbb{8}$, either $A \cong$ $B\left(\mathbb{P}^{2}, \mathcal{O}(1), \sigma\right)$ for some $\sigma$-in which case Theorem 3.2 implies that $\operatorname{QGr}(A) \cong \mathbb{P}^{2}$ or $A$ has a normal element $g$ such that $A / g A$ is isomorphic to $B(E, \mathcal{L}, \sigma)$ for some elliptic curve $E$. In the latter case we call $\mathrm{QGr}(A)$ a nontrivial noncommutative $\mathbb{P}^{2}$.

We emphasize that this shows that $A$ is not determined by $\mathrm{QGr}(A)$. However, when one works with $\mathbb{Z}$-algebras, as we do below, this issue goes away since Corollary 11.2 .4 shows that the $\mathbb{Z}$-algebra associated to $\operatorname{QGr}(A)$ is determined, up to isomorphism, by that category.

One may be tempted to extend Definition 11.2.1 to say that a noncommutative weighted $\mathbb{P}^{2}$ is the category $\operatorname{QGr}(A)$, for an arbitrary AS regular algebra $A$ of dimension three. However, as we show in the next section, this is too restrictive. But, if that is the case, is the definition of a noncommutative $\mathbb{P}^{2}$ also too restrictive? As an indication that the definition is appropriate, we show that any possible deformation (we use the term only in an informal sense that will become clearer as we proceed) of $\mathbb{P}^{2}$ will fit into the above classification, and so this suggested definition of a noncommutative $\mathbb{P}^{2}$ is appropriate.

The approach we use for $\mathbb{P}^{2}$ was found by Bondal and Polishchuk in [19] and we give a slightly personalized interpretation of the material in that paper. Let $\mathcal{C}$ be our supposed noncommutative projective plane. The point of view of [19] is that a good definition for a noncommutative $\mathbb{P}^{2}$ should at least encompass deformations of $\mathrm{Q} \operatorname{ch}\left(\mathbb{P}^{2}\right)$ as a category. One way to control deformations of commutative schemes is through the structure of their exceptional objects. Recall that an object $E$ in an abelian category is exceptional if it satisfies

$$
\operatorname{Ext}^{i}(E, E)= \begin{cases}k & \text { if } i=0 \\ 0 & \text { otherwise. }\end{cases}
$$

Standard deformation theory yields that exceptional objects lift uniquely to a deformation.

On $\mathbb{P}^{2}$ the line bundles $\mathcal{O}_{\mathbb{P}^{2}}(n)$ are clearly exceptional objects and so it is reasonable to impose the existence of analogous objects $\mathcal{O}(n)$ in $\mathcal{C}$. Now the ampleness of a sequence is also stable under deformation [88, so we will assume that the $\mathcal{O}(n)$ in fact form an ample sequence in $\mathcal{C}$. By Theorem 11.1.1, $\mathcal{C}$ will be equivalent to $\mathrm{Q} \operatorname{Gr}(A)$ where $A$ is the $\mathbb{Z}$-graded algebra defined by $A=\bigoplus_{j \geq i} A_{i j}$ with $A_{i j}=\operatorname{Hom}(\mathcal{O}(-j), \mathcal{O}(-i))$. Note in passing that we do not have a natural $\mathbb{N}$-graded algebra associated to our data. The problem is that if we, say, took $A^{\prime}=\bigoplus_{i \geq 0} \operatorname{Hom}(\mathcal{O}(0), \mathcal{O}(-i))$, then we have no convenient shift functor and so it is unclear how one defines the multiplication on $A^{\prime}$.

To conclude we have to describe $A$ more precisely. The main idea is that the dimension of $\operatorname{Ext}_{\mathcal{C}}^{t}(\mathcal{O}(-j), \mathcal{O}(-i))$ may decrease under deformation but the Euler form

$$
\langle\mathcal{O}(-j), \mathcal{O}(-i)\rangle=\sum_{t}(-1)^{t} \operatorname{dim} \operatorname{Ext}^{t}(\mathcal{O}(-j), \mathcal{O}(-i))
$$

should remain constant. This allows us to compute $\operatorname{dim} A_{i j}$ by using the fact that $\operatorname{Ext}_{\mathcal{O}^{2}}^{t}\left(\mathcal{O}_{\mathbb{P}^{2}}(-j), \mathcal{O}_{\mathbb{P}^{2}}(-i)\right)=0$ for $t>0$ and $j \geq i$. Putting this together with 
a little linear algebra shows that $A$ must satisfy the properties of the following definition:

Definition 11.2.2. A (noncommutative) Bondal-Polishchuk $\mathbb{P}^{2}$ is an abelian category of the form $\mathrm{QGr}(A)$ where $A$ is a $\mathbb{Z}$-algebra with the following properties:

(P1) $A$ is generated by the $A_{i, i+1}$.

(P2) The following holds for the dimensions of $A_{i j}$ :

$$
\operatorname{dim} A_{i, i+n}= \begin{cases}(n+1)(n+2) / 2 & \text { if } n \geq 0 \\ 0 & \text { otherwise }\end{cases}
$$

(P3) Put $V_{i}=A_{i, i+1}$. By (P2), the multiplication map $V_{i} \otimes V_{i+1} \rightarrow A_{i, i+2}$ has a 3-dimensional kernel $R_{i}$. We require that the $R_{i}$ generate the relations of $A$.

(P4) Put $W_{i}=V_{i} \otimes R_{i+1} \cap R_{i} \otimes V_{i+2}$ inside $V_{i} \otimes V_{i+1} \otimes V_{i+2}$. Dimension counting using (P2) and (P3) reveals that $\operatorname{dim} W_{i}=1$. If $W_{i}=w_{i} k$, we require that $w_{i}$ is a non-degenerate tensor, both as an element of $V_{i} \otimes R_{i+1}$ and as an element of $R_{i} \otimes V_{i+2}$.

It is easily seen that if $A$ is a three dimensional AS regular algebra with Hilbert series $(1-t)^{-3}$ and associated $\mathbb{Z}$-algebra $A^{\prime}$, then $A^{\prime}$ satisfies the above axioms.

In 19 the authors classify the $\mathbb{Z}$-algebras satisfying $(\mathrm{P} 1)-(\mathrm{P} 4)$ in terms of a triple $\left(Y, \mathcal{L}_{1}, \mathcal{L}_{2}\right)$, where $Y$ is either $\mathbb{P}^{2}$ or an elliptic curve therein and the $\mathcal{L}_{i}$ are ample line bundles on $Y$ satisfying certain technical conditions. These imply that $\mathcal{L}_{2}=\mathcal{L}_{1}^{\sigma}$ for an automorphism $\sigma$ on $Y$. Thus it is not surprising that they further obtain:

Theorem 11.2.3. Assume that char $k \neq 3$. Then the noncommutative BondalPolishchuk $\mathbb{P}^{2}$ 's are exactly the noncommutative $\mathbb{P}^{2}$ 's. More precisely, every $\mathbb{Z}$ algebra satisfying (P1)-(P4) is isomorphic to a $\mathbb{Z}$-algebra obtained from a connected graded AS algebra of dimension three with Hilbert series $(1-t)^{-3}$.

This theorem provides another justification for our definition of a noncommutative $\mathbb{P}^{2}$. In [19] it was only stated in characteristic zero.

If $A$ is a $\mathbb{Z}$-algebra and $m \in \mathbb{Z}$, then we may define the shifted $\mathbb{Z}$-algebra $A(m)$ by $A(m)_{i j}=A(m)_{i+m, j+m}$. Clearly $\operatorname{Gr}(A(m)) \cong \operatorname{Gr}(A)$ and $\operatorname{QGr}(A(m)) \cong \operatorname{QGr}(A)$ but $A$ comes from a graded ring if and only if $A \cong A(1)$. If $\mathcal{C}$ is a noncommutative Bondal-Polishchuk $\mathbb{P}^{2}$, then it is easy to see that the sequence of objects $(\mathcal{O}(n))_{n}$ is determined up to shift by the categorical properties of $\mathcal{C}$ and hence that the associated $\mathbb{Z}$-algebra is also determined up to shifting (see [88] for the details). Combined with Theorem 11.2.3 these observations prove:

Corollary 11.2.4. Assume that char $k \neq 3$. If $\mathcal{C}$ is a noncommutative $\mathbb{P}^{2}$, then the associated $\mathbb{Z}$-algebra $A$ is unique. For example, if $\mathcal{C}=\mathrm{Qch}\left(\mathbb{P}^{2}\right)$, then $A$ is just the $\mathbb{Z}$-algebra associated with $k[x, y, z]$.

11.3. Noncommutative quadrics. Even if one restricts to algebras generated in degree one, there are two classes of Artin-Schelter regular algebras of dimension three: the ones already mentioned with Hilbert series $(1-t)^{-3}$ and a second class $\mathcal{Q}$ of algebras that require just two generators and have the Hilbert series $(1-$ $t)^{-2}\left(1-t^{2}\right)^{-1}$ of a weighted polynomial ring $k\left[u_{1}, u_{2}, u_{3}\right]$ with weights $(1,1,2)$. The archetypal example of this second class is the enveloping algebra $U(\mathfrak{g})$ of the three-dimensional Heisenberg Lie algebra $\mathfrak{g}$; thus $\mathfrak{g}$ has basis $x, y, z$ with relations $[x y]=z,[x z]=0=[y z]$. Clearly $U(\mathfrak{g})$ is generated by $x$ and $y$, and if we assign 
$x, y, z$ weights $1,1,2$, then the Poincaré-Birkoff-Witt Theorem ensures that $U(\mathfrak{g})$ is a graded ring of the required Hilbert series. Given the results from the last section, one might propose that a noncommutative quadric surface be defined as $\operatorname{QGr}(A)$ for any $A \in \mathcal{Q}$. (These surfaces are smooth-see Remark [2.1.3])

Unfortunately, this is inadequate since there appear to be far more noncommutative quadrics than AS regular rings. For example, the Hilbert series of the factor $\operatorname{ring} R_{\lambda}=R_{\lambda}(E, \mathcal{L}, \sigma)$ of the 4 dimensional Sklyanin algebra, as described in Example 8.5, is the same as that of the homogeneous coordinate ring of a quadric in $\mathbb{P}^{3}$. Therefore we would like to consider $R_{\lambda}$ as a noncommutative quadric (see below and $₫ 12$ for a more formal justification). But $R_{\lambda}$ depends upon 3 paramenters (one each for $E, \mathcal{L}, \sigma$ and $\lambda$ minus one for the automorphism group of $E$ ) whereas a similar count reveals that the AS regular rings depend on just 2 .

In order to get an idea about how many noncommutative quadrics one should expect, we follow the Bondal-Polishchuk approach from the last section, but with $\mathbb{P}^{2}$ replaced by $\mathbb{P}^{1} \times \mathbb{P}^{1}$. For this variety some of the exceptional line bundles are:

$$
\mathcal{O}(n)= \begin{cases}\mathcal{O}(k, k) & \text { if } n=2 k \\ \mathcal{O}(k, k+1) & \text { if } n=2 k+1\end{cases}
$$

where $\mathcal{O}(a, b)=\mathcal{O}_{\mathbb{P}^{1}}(a) \otimes \mathcal{O}_{\mathbb{P}^{1}}(b)$. If one now follows the approach outlined in the last section one arrives at:

Definition 11.3.1. 88 A noncommutative quadric is a Grothendieck category of the form $\mathrm{QGr}(A)$ where $A$ is a $\mathbb{Z}$-algebra satisfying the following properties.

(Q1) $A$ is generated by the $A_{i, i+1}$.

(Q2) The following holds for the dimensions of $A_{i j}$ :

$$
\operatorname{dim} A_{i, i+n}= \begin{cases}0 & \text { if } n<0 \\ (k+1)^{2} & \text { if } n=2 k \text { and } n \geq 0 \\ (k+1)(k+2) & \text { if } n=2 k+1 \text { and } n \geq 0 .\end{cases}
$$

(Q3) Put $V_{i}=A_{i, i+1}$. By (Q2), $A_{i, i+2}=V_{i} \otimes V_{i+1}$ and the multiplication map $V_{i} \otimes V_{i+1} \otimes V_{i+2} \rightarrow A_{i, i+3}$ has two-dimensional kernel $R_{i}$. We require that the $R_{i}$ generate the relations of $A$.

(Q4) Put $W_{i}=V_{i} \otimes R_{i+1} \cap R_{i} \otimes V_{i+3}$ inside $V_{i} \otimes V_{i+1} \otimes V_{i+2} \otimes V_{i+3}$. Dimension counting using (Q2) and (Q3) reveals that $\operatorname{dim} W_{i}=1$. If $W_{i}=w_{i} k$, we require that $w_{i}$ is a non-degenerate tensor, both as an element of $V_{i} \otimes R_{i+1}$ and as an element of $R_{i} \otimes V_{i+3}$.

As yet there is no analogue of Theorem 11.2 .3 but the $\mathbb{Z}$-algebras satisfying (Q1)-(Q4) are surely more general than the AS-regular algebras $A \in \mathcal{Q}$, simply because they depend on one more parameter. In more detail, the classification in 88] of $\mathbb{Z}$-algebras satisfying (Q1)-(Q4) is in terms of quadruples $\left(Y, \mathcal{L}_{0}, \mathcal{L}_{1}, \mathcal{L}_{2}\right)$ where either:

1. $\left(Y, \mathcal{L}_{0}, \mathcal{L}_{1}, \mathcal{L}_{2}\right)=\left(\mathbb{P}^{1} \times \mathbb{P}^{1}, \mathcal{O}(1,0), \mathcal{O}(0,1), \mathcal{O}(1,0)\right)$; or else

2. (a) $Y$ is a curve which is embedded as a divisor of degree $(2,2)$ in $\mathbb{P}^{1} \times \mathbb{P}^{1}$ by the global sections of both $\left(\mathcal{L}_{0}, \mathcal{L}_{1}\right)$ and $\left(\mathcal{L}_{1}, \mathcal{L}_{2}\right)$.

(b) $\mathcal{L}_{0} \approx \mathcal{L}_{2}$.

(c) $\operatorname{deg}\left(\mathcal{L}_{0} \mid C\right)=\operatorname{deg}\left(\mathcal{L}_{2} \mid C\right)$ for every irreducible component $C$ of $Y$.

The construction of the quadruple associated to a $\mathbb{Z}$-algebra $A$ is similar to the Bondal-Polishchuk approach in [19]: we consider $R_{0}$ as the equations for a closed 
subscheme $Y$ of $\mathbb{P}\left(V_{0}^{*}\right) \times \mathbb{P}\left(V_{1}^{*}\right) \times \mathbb{P}\left(V_{2}^{*}\right)$ and we put $\mathcal{L}_{i}=\operatorname{pr}_{i}^{*}(\mathcal{O}(1))$ where $\operatorname{pr}_{i}$ denotes the projection onto $\mathbb{P}\left(V_{i}^{*}\right)$. Conversely, to construct a $\mathbb{Z}$-algebra from a triple $\left(Y, \mathcal{L}_{0}, \mathcal{L}_{1}, \mathcal{L}_{2}\right)$, one may proceed as follows. First construct a sequence of line bundles (called an elliptic helix in [19]) $\left(\mathcal{L}_{i}\right)_{i}$ defined by the property

$$
\mathcal{L}_{i} \otimes \mathcal{L}_{i+1}^{-1} \otimes \mathcal{L}_{i+2}^{-1} \otimes \mathcal{L}_{i+3}=\mathcal{O}_{Y} .
$$

We put $V_{i}=\Gamma\left(Y, \mathcal{L}_{i}\right)$ and let $R_{i}$ be the kernel of the canonical map

$$
\Gamma\left(Y, \mathcal{L}_{i}\right) \otimes \Gamma\left(Y, \mathcal{L}_{i+1}\right) \otimes \Gamma\left(Y, \mathcal{L}_{i+2}\right) \rightarrow \Gamma\left(Y, \mathcal{L}_{i} \otimes \mathcal{L}_{i+1} \otimes \mathcal{L}_{i+2}\right) .
$$

Finally, $A$ is defined to be the $\mathbb{Z}$-algebra freely generated by the $V_{i}$, subject to the relations $R_{i}$.

An interesting open question is to determine when $\mathrm{QGr}(A) \cong \mathrm{QGr}\left(A^{\prime}\right)$ for $\mathbb{Z}$ algebras satisfying (Q1)-(Q4). This is even unanswered for the factor rings $R_{\lambda}=$ $\mathrm{Skl}_{4} /\left(\lambda_{1} \Omega_{1}+\lambda_{2} \Omega_{2}\right)$ of the Sklyanin algebra $\mathrm{Skl}_{4}$ described in Example 8.5. (As we will see in the next section, generically these rings do define quadrics.) There is however a reasonable guess as to the correct answer that goes back to the translation principle for simple Lie algebras. For simplicity assume that $k=\mathbb{C}$. Then $\mathrm{Skl}_{4}$ can be thought of as a deformation of the homogenized enveloping algebra of the complex Lie algebra $\mathfrak{s l}(2)$ (this is the ring obtained by taking $\alpha_{i}=0$ for all $i$ in the definition of $\left.\mathrm{Skl}_{4}\right)$. We want to regard $\mathrm{Skl}_{4}$ as a deformation of $U(\mathfrak{s l}(2))$ since then the $R_{\lambda}$ correspond to the infinite dimensional primitive factors $U_{\mu}=U(\mathfrak{s l}(2)) /(\Omega-\mu)$, where $\Omega$ is the Casimir element and $\mu \in k$. The translation principle [15] asserts that certain $U_{\mu}$ are Morita equivalent and by [27] there are no other Morita equivalences.

Much of this generalizes. Notably, there exists an analogous translation principle for $\mathrm{Skl}_{4}$ (see [86]) and for noncommutative quadrics (see 88]). The translation principle for noncommutative quadrics comes out of the observation that, in contrast to Corollary 11.2.4, the sequence $(\mathcal{O}(n))_{n}$ is not now determined up to shift. For example, on $\mathbb{P}^{1} \times \mathbb{P}^{1}$ we could equally well have chosen

$$
\mathcal{O}(n)= \begin{cases}\mathcal{O}(k, k+a) & \text { if } n=2 k \\ \mathcal{O}(k, k+1+a) & \text { if } n=2 k+1\end{cases}
$$

in place of (11.2). Analyzing the possible sequences $(\mathcal{O}(n))_{n}$ leads to the desired translation principle. The analogue of Hodges' theorem, which asserts that there are no other Morita equivalences, is also likely to be true. This has been proved if the triple $\left(Y, \mathcal{L}_{0}, \mathcal{L}_{1}, \mathcal{L}_{2}\right)$ is sufficiently generic, but in the general case a few technical problems remain which the second author is currently trying to solve.

\section{2. $\mathbb{P}^{1}$-BUNDLES}

A question that was not explicitly discussed in the last section is whether the quadratic quotients $R_{\lambda}$ of the Sklyanin algebra $\mathrm{Skl}_{4}$ do in fact correspond to noncommutative quadrics. One way to tackle this is through the concept of a $\mathbb{P}^{1}$ bundle. Recall that commutative quadrics can be written as a $\mathbb{P}^{1}$-bundle $\mathbb{P}(\mathcal{E})$ where $\mathcal{E}=\mathcal{O}_{\mathbb{P}^{1}}^{2}$ [26, $\left.\S I I .7\right]$. In this section we give similar results for noncommutative quadrics (see Theorem 12.6) and for the $R_{\lambda}$ (Proposition 12.2). This shows that, generically, the $R_{\lambda}$ are noncommutative quadrics.

In order to explain this material we have to introduce some new concepts. Some of these definitions are a little technical, but the reader will not go far wrong if, initially, he/she thinks of bimodules in the more mundane, ring theoretic sense. 
Below $W, X, Y, Z$ are schemes of finite type over $k$. A coherent $\mathcal{O}_{X}-\mathcal{O}_{Y}$-bimodule $\mathcal{E}$ is by definition a coherent $\mathcal{O}_{X \times Y}$-module such that the support of $\mathcal{E}$ is finite over both $X$ and $Y$. We denote the abelian category of coherent $\mathcal{O}_{X}-\mathcal{O}_{Y}$-bimodules by $\operatorname{bimod}\left(\mathcal{O}_{X}-\mathcal{O}_{Y}\right)$. Any coherent bimodule $\mathcal{E}$ defines a right exact functor $-\otimes_{\mathcal{O}_{X}} \mathcal{E}$ : $\mathrm{Qch}(X) \rightarrow \mathrm{Q} \operatorname{ch}(Y)$ via $\operatorname{pr}_{2 *}\left(\operatorname{pr}_{1}^{*}(-) \otimes_{\mathcal{O}_{X \times Y}} \mathcal{E}\right)$. Similarly, if $\mathcal{F}$ is a coherent $\mathcal{O}_{Y^{-}} \mathcal{O}_{Z^{-}}$ bimodule, then the tensor product $\mathcal{E} \otimes_{\mathcal{O}_{Y}} \mathcal{F}$ is defined as $\operatorname{pr}_{13 *}\left(\operatorname{pr}_{12}^{*} \mathcal{E} \otimes_{\mathcal{O}_{X \times Y \times Z}}\right.$ $\left.\operatorname{pr}_{23}^{*} \mathcal{F}\right)$. It is easy to show that this definition yields all the expected properties.

Now assume that we have finite maps $\alpha: W \rightarrow X$ and $\beta: W \rightarrow Y$. If $\mathcal{H}$ is a coherent $\mathcal{O}_{W}$-module, then we denote the $\mathcal{O}_{X}-\mathcal{O}_{Y}$-bimodule $(\alpha, \beta)_{*} \mathcal{H}$ by ${ }_{\alpha} \mathcal{H}_{\beta}$. Any bimodule $\mathcal{E}$ can be presented in this form since we may take $W$ to be the support of $\mathcal{E}$. From the definition it is easy to check that $-\otimes_{\alpha} \mathcal{H}_{\beta}=\beta_{*}\left(\alpha^{*}(-) \otimes_{\mathcal{O}_{W}} \mathcal{H}\right)$.

Example 12.1. Let $\mathcal{L}$ be a line bundle on $X$ and let $\sigma \in \operatorname{Aut}(X)$. Put $\mathcal{M}={ }_{\text {id }} \mathcal{L}_{\sigma}$. Then we obtain $-\otimes_{\mathcal{O}_{X}} \mathcal{M}=\sigma_{*}\left(-\otimes_{\mathcal{O}_{X}} \mathcal{L}\right)$. Thus $-\otimes_{\mathcal{O}_{X}} \mathcal{M}$ is the standard autoequivalence of $\mathrm{Q} \operatorname{ch}(X)$ that was introduced in $\$ 3$ and played such a fundamental role in the construction of twisted homogeneous coordinate rings.

We will define graded algebras in $\operatorname{bimod}\left(\mathcal{O}_{X}-\mathcal{O}_{X}\right)$ as formal direct sums $\mathcal{A}=$ $\bigoplus \mathcal{A}_{n}$, for $\mathcal{A}_{n} \in \operatorname{bimod}\left(\mathcal{O}_{X}-\mathcal{O}_{X}\right)$, equipped with multiplication maps $\mathcal{A}_{m} \otimes_{\mathcal{O}_{X}}$ $\mathcal{A}_{n} \rightarrow \mathcal{A}_{m+n}$ and a unit map $\mathcal{O}_{X} \rightarrow \mathcal{A}_{0}$ satisfying the usual properties. A graded right $\mathcal{A}$ module is a formal direct sum $\mathcal{M}=\bigoplus_{n} \mathcal{M}_{n}$, for $\mathcal{M}_{n} \in \mathrm{Q} \operatorname{ch}(X)$, equipped with multiplication maps $\mathcal{M}_{m} \otimes_{\mathcal{O}_{X}} \mathcal{A}_{n} \rightarrow \mathcal{M}_{m+n}$, again satisfying the usual properties. We denote the corresponding category by $\operatorname{Gr}(\mathcal{A})$. The quotient category $\operatorname{QGr}(\mathcal{A})$ is defined in the expected way, and other constructions like $\mathbb{Z}$-algebras generalize routinely to this context. The formal definitions can be found in 87].

We now turn to the four dimensional Sklyanin algebra $\mathrm{Skl}_{4}=\operatorname{Skl}_{4}(E, \mathcal{L}, \sigma)$ and its factors $R_{\lambda}(E, \mathcal{L}, \sigma)=\operatorname{Skl}_{4} /\left(\Omega_{\lambda}\right)$, for $\lambda \in \mathbb{P}^{1}$, as defined in Example 8.5. Fix a map $\omega: E \rightarrow \mathbb{P}^{1}$ of degree two.

Proposition 12.2. [86, Theorem 7.2.1] Let $R_{\lambda}=R_{\lambda}(E, \mathcal{L}, \sigma)$ and assume that $|\sigma|=\infty$. Then, for sufficiently general $\lambda$, we have $\mathrm{QGr}\left(R_{\lambda}\right)=\operatorname{QGr}(T \mathcal{E} /(Q))$ where $\mathcal{E}={ }_{\omega} \mathcal{V}_{\omega \sigma}$ for some line bundle $\mathcal{V}=\mathcal{V}(\lambda)$ of degree four on $E, T \mathcal{E}$ is the tensor algebra of $\mathcal{E}$ and $Q$ is an invertible subbimodule of $\mathcal{E} \otimes \mathcal{E}$.

Since $\mathcal{E}$ is locally free of rank two on both sides we may clearly view $\mathrm{Q} \operatorname{Gr}(T \mathcal{E} /(Q))$ as a noncommutative analogue of $\mathbb{P}(\mathcal{E})$. We have therefore written $\mathrm{Q} \operatorname{Gr}\left(R_{\lambda}\right)$ as a noncommutative analogue of a $\mathbb{P}^{1}$-bundle on $\mathbb{P}^{1}$.

An obvious question raised by Proposition 12.2 is to determine when it is possible to form sheaves of algebras of the form $T \mathcal{E} / Q$, where $\mathcal{E}$ is a rank two locally free $\mathcal{O}_{X}$-bimodule $\mathcal{E}$ over a smooth curve $X$ and $Q$ is an appropriate subbimodule of $\mathcal{E} \otimes \mathcal{E}$. One solution is given by Patrick in [54, [55]. In the commutative case, $Q$ is just the subbimodule of $\mathcal{E} \otimes \mathcal{E}$ generated by sections of the form $x \otimes y-y \otimes x$. Thus, in general, one at least needs $Q$ to be a rank one subbimodule of $\mathcal{E} \otimes \mathcal{E}$ that cannot be decomposed as $Q_{1} \otimes Q_{2}$ for $Q_{i} \subset \mathcal{E}$. This puts severe restrictions on the possible choices of $\mathcal{E}$, to the extent that Patrick can classify them into just four basic classes [54].

This suggests that $T \mathcal{E} / Q$ may be the wrong model for $\mathbb{P}(\mathcal{E})$. As the second author has recently observed, working in the more general setting of $\mathbb{Z}$-algebras allows one to form an appropriate analogue of $\mathbb{P}(\mathcal{E})$ without any such restrictions on the rank two bimodule $\mathcal{E}$. This even enables one to define the categories $\operatorname{Gr}(T \mathcal{E} /(Q))$ and 
QGr $(T \mathcal{E} /(Q))$ from Proposition 12.2 independently of $Q$. We briefly sketch the construction.

Assume that $X$ and $Y$ are smooth schemes and let $\mathcal{E} \in \operatorname{bimod}\left(\mathcal{O}_{X}-\mathcal{O}_{Y}\right)$ be locally free of rank two on both sides. Then it is easy to show that $-\otimes_{\mathcal{O}_{X}} \mathcal{E}$ has a right adjoint $-\otimes_{\mathcal{O}_{Y}} \mathcal{E}^{*}$, where $\mathcal{E}^{*} \in \operatorname{bimod}\left(\mathcal{O}_{Y}-\mathcal{O}_{X}\right)$ is also locally free of rank two on both sides. Repeating this construction, we may define $\mathcal{E}^{* 2}=\mathcal{E}^{* *}$ by requiring that $-\otimes_{\mathcal{O}_{X}} \mathcal{E}^{* *}$ is the right adjoint of $-\otimes_{\mathcal{O}_{Y}} \mathcal{E}^{*}$. By induction, we define $\mathcal{E}^{* n}=\left(\mathcal{E}^{*(n-1)}\right)^{*}$ for $n \geq 0$ and by considering left adjoints we may define $\mathcal{E}^{* n}$ for $n<0$. Standard properties of adjoint functors yield a bimodule inclusion $j_{n}: \mathcal{O}_{X_{n}} \hookrightarrow \mathcal{E}^{* n} \otimes_{\mathcal{O}_{X_{n+1}}} \mathcal{E}^{*(n+1)}$, where $X_{n}$ is respectively $X$ or $Y$ depending on whether $n$ is even or odd.

We now define $S \mathcal{E}$ as the $\mathbb{Z}$-algebra which satisfies

(a) $(S \mathcal{E})_{n n}=\mathcal{O}_{X_{n}}$;

(b) $(S \mathcal{E})_{n, n+1}=\mathcal{E}^{* n}$;

(c) $S \mathcal{E}$ is freely generated by the $(S \mathcal{E})_{n, n+1}$, subject to the relations given by the images of $j_{n}$.

It is easy to show:

Proposition 12.3. Let $X=Y=E$ and $\mathcal{E}$ be defined by Proposition 12.2. Then the category $\operatorname{Gr}(T \mathcal{E} /(Q))$ which appeared in Proposition 12.2 is equivalent to $\operatorname{Gr}(S \mathcal{E})$. Thus the construction is independent of $Q$ !

Given the success of this approach, we propose the following definition.

Definition 12.4. A noncommutative $\mathbb{P}^{1}$ bundle on a smooth scheme $X$ of finite type over $k$ is a category of the form $\mathrm{QGr}(S \mathcal{E})$ where $\mathcal{E} \in \operatorname{bimod}\left(\mathcal{O}_{X}-\mathcal{O}_{Y}\right)$, for some smooth scheme $Y$, such that $\mathcal{E}$ is locally free of rank two on both sides.

We leave it to the reader to check the following result.

Proposition 12.5. Let $\mathcal{E}$ be as in Definition 12.4 and assume that $\mathcal{U}, \mathcal{V}$ are line bundles on $X$ and $Y$ respectively. Put $\mathcal{F}=\mathcal{U} \otimes_{\mathcal{O}_{X}} \mathcal{E} \otimes_{\mathcal{O}_{Y}} \mathcal{V}$. Then $\operatorname{Gr}(S \mathcal{E})$ and $\operatorname{Gr}(S \mathcal{F})$ are equivalent.

Finally we mention the following result from 88 .

Theorem 12.6. Assume that $A$ is a $\mathbb{Z}$-algebra satisfying (Q1)-(Q4) from (11.3.1) with associated quadruple $\left(Y, \mathcal{L}_{0}, \mathcal{L}_{1}, \mathcal{L}_{2}\right)$. Assume that, in the corresponding sequence $\left(Y,\left(\mathcal{L}_{i}\right)_{i}\right)$ defined by (11.3), we have $\mathcal{L}_{0} \neq \mathcal{L}_{2 n+1}$ for $n \geq 0$. Let $Y^{\prime}$ be the image of $Y$ in $\mathbb{P}^{1} \times \mathbb{P}^{1}$ under the map defined by $\left(\mathcal{L}_{0}, \mathcal{L}_{2}\right)$ and let $\mathcal{L}_{1}^{\prime}$ be the direct image of $\mathcal{L}_{1}$ under the induced map $Y \rightarrow Y^{\prime}$. Then $\mathrm{QGr}(A)=\mathrm{Q} \operatorname{Gr}(S \mathcal{M})$ for $\mathcal{M}={ }_{\mathrm{pr}_{1}}\left(\mathcal{L}_{1}^{\prime}\right)_{\mathrm{pr}_{2}}$.

Combining (12.2 12.6) shows that the quotients $R_{\lambda}$ of the four-dimensional Sklyanin algebras are noncommutative quadrics.

\section{Noncommutative Blowing uP}

In this section we summarize some recent results on blowing up of noncommutative surfaces. In particular, this allows us to give noncommutative analogues of the construction of cubic surfaces, Cremona transformation and quadrics that were outlined in $\S \$ 9.2$ 9.4, as well as answer the question posed by Example10.1.1. This material is all taken from [87], [89], to which the reader is referred for more details. 
13.1. Motivation, definition and properties. To fix ideas let us first consider the affine case. Assume that $R$ is a finitely generated noetherian $k$-algebra and let $\mathfrak{m}$ be a maximal ideal of $R$ such that $R / \mathfrak{m}=k$. Then, as in (9.1), a natural idea is to define the blow-up of $R$ at $\mathfrak{m}$ as $\operatorname{QGr}(D)$ where $D=R \oplus \mathfrak{m} \oplus \mathfrak{m}^{2} \oplus \mathfrak{m}^{3} \oplus \cdots$ is the Rees algebra associated to $\mathfrak{m}$. Unfortunately this naïve definition is often faulty, as is shown by the following example from [2].

Let $R=k\{x, y\} /(y x-x y-y)$ with $\mathfrak{m}=(x, y)$. Thus $R$ is the enveloping algebra of the two-dimensional solvable Lie algebra and resembles the polynomial ring in two variables that was considered in $\$ 9.1$. However, $\mathfrak{m}^{n}=\left(x^{n}, y\right)$. Hence the analogue of the exceptional curve

$$
D / \mathfrak{m} D=R / \mathfrak{m} \oplus \mathfrak{m} / \mathfrak{m}^{2} \oplus \mathfrak{m}^{2} / \mathfrak{m}^{3} \oplus \cdots
$$

is isomorphic to $k[x]$. Thus $\mathrm{QGr}(D / \mathfrak{m})$ is a point, whereas intuitively we would expect it to be one-dimensional.

What has gone wrong is that, unlike the commutative case, $\mathfrak{m}$ is "linked" to other prime ideals in the sense that there are other simple modules $R / \mathfrak{m}_{i}$ that appear in the injective hull $E\left({ }_{R} R / \mathfrak{m}\right)$. Somehow, we need to involve them in the definition of blowing-up. A general discussion of linked primes can be found in 24 Chapters 11,12] (see, in particular [24, Example 11G] for a discussion of the ring $R$ ), but we will use a simpler approach for this example. The basic observation is that the commutator ideal of $R$ is $y R$ and the element $y$ induces an automorphism $\tau$ of $R$ by $a \mapsto y^{-1} a y$. The prime ideals $R / \mathfrak{m}_{i}$ mentioned above are exactly the $\tau^{i}(\mathfrak{m})$. The correct blow-up of $R$ at $\mathfrak{m}$ turns out to be $\mathrm{QGr}(D)$, for

$$
D=R \oplus I_{1} \oplus I_{2} \oplus I_{3} \oplus \cdots \quad \text { where } \quad I_{n}=\mathfrak{m} \tau(\mathfrak{m}) \cdots \tau^{n-1}(\mathfrak{m})_{\tau^{n}}
$$

(thus, the right action of $R$ on $I_{n}$ is twisted by $\tau^{n}$ ). If $L=D / \tau^{-1}(\mathfrak{m}) D$, then $\operatorname{dim} L_{u}=u+1$ and so $L$ plays the role of the exceptional curve. We refer the reader to [2] for a detailed workout of this example.

This construction generalizes to any ring whose commutator ideal is invertible. The latter hypotheses is not unreasonable since, if we look at the case of a Poisson surface, we see that we expect a noncommutative smooth surface to contain a commutative curve.

However, we also want blow-ups to apply in more general situations where the concept of the commutator ideal does not really make sense, in particular since we want to blow up at one-sided ideals. One such situation is given by the noncommutative $\mathbb{P}^{2}$ 's and $\mathbb{P}^{1} \times \mathbb{P}^{1}$ 's which were introduced in $₫ 11$. For simplicity let us consider the nontrivial noncommutative $\mathbb{P}^{2}$ 's, as introduced in Definition 11.2.1 By construction, such a category $\operatorname{QGr}(A)$ contains a subcategory isomorphic to $\mathrm{Q} \operatorname{ch}(E)$, for some elliptic curve $E$ and so, as in 98 , we consider $E$ to be a closed (commutative) subscheme of the noncommutative scheme QGr( $A)$.

Now let $p \in E$. The previous discussion suggests that it should be possible to blow up $p$. However it is not clear how to proceed. As explained in 8,8 , corresponds to a point module over $A$ which, since it is only a right module, cannot be used to construct a Rees algebra.

Such difficulties can be solved by working with categories rather than algebras. To explain this, we need a little more geometric language. As before, we define a noncommutative scheme to be a Grothendieck category, but to stress the geometric viewpoint we will now use roman capitals $X, Y, \ldots$ If $X$ is a commutative scheme, then we will identify $X$ with $\mathrm{Q} \operatorname{ch}(X)$. This is justified by the reconstruction theorem 
proved by Rosenberg 61. A morphism $\alpha: X \rightarrow Y$ between noncommutative schemes will be a right exact functor $\alpha^{*}: Y \rightarrow X$ possessing a right adjoint (denoted by $\alpha_{*}$ ). In this way the noncommutative schemes form a category (more precisely a two-category; see [37]).

Let $X, Y, Z, \ldots$ be noncommutative schemes. Inspired by $\$ 12$ we would like to introduce $X-Y$-bimodules. Unfortunately the definition given in $\$ 12$ does not generalize to the noncommutative case. Therefore we change the definition of bimodules: An $X-Y$ bimodule is just an object in

$$
\operatorname{Bimod}(X-Y)=\{\text { morphisms } X \rightarrow Y\}
$$

We write the functor corresponding to $\mathcal{M} \in \operatorname{Bimod}(X-Y)$ as $-\otimes_{X} \mathcal{M}$. Likewise we write composition of functors

$$
\operatorname{Bimod}(X-Y) \times \operatorname{Bimod}(Y-Z) \rightarrow \operatorname{Bimod}(X-Z)
$$

as $-\otimes_{Y}-$. It is clear that $\operatorname{Bimod}(X-X)$ contains a canonical object $o_{X}$ given by the identity functor.

We will need to talk about images and subobjects in $\operatorname{Bimod}(X-Y)$, which is a problem since $\operatorname{Bimod}(X-Y)$ is not an abelian category (at least not in any obvious way). We resolve the problem by embedding $\operatorname{Bimod}(X-Y)$ into the opposite category to the category of left exact functors from $Y$ to $X$. As this bigger category is abelian, images and subobjects do exist there.

As $\operatorname{Bimod}(X-X)$ is a monoidal category we can define graded algebras and graded modules over it in formally the same way as this was done in $\$ 12$.

The definition of blowing-up given in 87] works in the situation where $X$ contains a commutative curve as a divisor. To make this more precise, we consider the identity functor $o_{X}$ on $X$, as defined above. It is clear that $o_{X}$ is an algebra and, furthermore, that $\operatorname{Mod}\left(o_{X}\right)=X$. We will assume that $o_{X}$ contains an invertible subbimodule $o_{X}(-Y)$ (an invertible ideal) such that $\operatorname{Mod}\left(o_{X} / o_{X}(-Y)\right)$ is equivalent to $\mathrm{Qch}(Y)$, for some commutative curve $Y$. We also need some sort of smoothness condition on $X$. Since it is obviously sufficient to impose this in a neighbourhood of $Y$, we assume that every object in $\mathrm{Q} c h(Y)$ has finite injective dimension in $X$. Finally we will assume that $X$ is noetherian, by which we mean that $X$ is a locally noetherian category.

We emphasize that all these assumptions hold when $X=\mathrm{QGr}(A)$ is a nontrivial noncommutative $\mathbb{P}^{2}$, with $Y=E$ the corresponding elliptic curve.

Let $p \in Y$. Then $p$ defines a subbimodule $m_{p}$ of $o_{X}$ which is the analogue of the maximal ideal corresponding to $p$ in $\mathcal{O}_{X}$, if $X$ were commutative. We put $I=m_{p} o_{X}(Y)$ and define

$$
\mathcal{D}=o_{X} \oplus I \oplus I^{2} \oplus \cdots
$$

where $I^{n}$ is defined as the image of $I^{\otimes n}$ in $o_{X}(n Y) \stackrel{\text { def }}{=} o_{X}(-Y)^{\otimes-n}$. This is the Rees algebra associated to $I$ and we define the blow-up $\widetilde{X}$ of $X$ in $p$ to be $\operatorname{QGr}(\mathcal{D})$.

A large part of [87] is devoted to proving that $\widetilde{X}$ satisfies properties similar to those of $X$ and, furthermore, that we have obtained an analogue of the blow-up of a commutative surface. In particular, that paper constructs a commutative diagram 
of noncommutative noetherian schemes

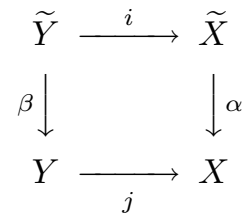

where the horizontal arrows are inclusions, $\widetilde{X}$ is again a noncommutative noetherian scheme and $\widetilde{Y}$ is a commutative curve which plays the role of the strict transform of $Y$. Moreover, $\widetilde{Y}$ is again a divisor in $\widetilde{X}$ and every object in $\operatorname{Qch}(\widetilde{Y})$ has finite injective dimension in $\widetilde{X}$.

13.2. More general birational transformations. As indicated in 99.5 it is convenient to have available more general birational transformations that accomplish combinations of blowing-ups and blowing-downs. It turns out that we can mimic the constructions given in 99.5 in the generality of $\$ 13.1$.

First assume that $d$ is a divisor in $o_{Y}$ and let $m_{Y, d}$ be the corresponding ideal in $o_{Y}$. Let $m_{d}$ be the inverse image of $m_{Y, d}$ in $o_{X}$. If we assume that we have a sequence $\left(\mathcal{L}_{n}\right)_{n}$ of noetherian objects on $X$, then we can define $\widetilde{X}$ as $\operatorname{QGr}(D)$, where $D$ is the $\mathbb{Z}$-algebra given by

$$
D_{a, b}=\operatorname{Hom}_{X}\left(\mathcal{L}_{-b}, \mathcal{L}_{-a} m_{\tau^{-a} d} \cdots m_{\tau^{-b+1} d}\right) .
$$

In this generality, presumably not much can be said about the properties of $\widetilde{X}$. However we can use these definitions to generalize the three classical examples given in $\$ 9.29 .4$.

13.3. The non-commutative Cremona transformation. Suppose that $X$ is a nontrivial noncommutative $\mathbb{P}^{2}$ (see Definition 11.2.1) and let $Y=E \subset X$ be the associated curve. Since $X=\mathrm{QGr}(A)$ for a graded ring $A$, there exist naturally defined objects $\mathcal{O}_{X}(n) \in X$, coming from the shifts $A(n)$. We put $\mathcal{L}_{n}=\mathcal{O}_{X}(2 n)$.

Choose three distinct non-collinear points $p, q, r \in Y$ and set $d=p+q+r$. Then it turns out that $\widetilde{X}$ as defined above is also a noncommutative $\mathbb{P}^{2}$. Using this we can show that there are exactly four different types of division algebras that can occur as the function field of a noncommutative $\mathbb{P}^{2}$, namely:

(a) Division rings finite dimensional over a commutative field of transcendence degree two.

(b) The quotient field of the first Weyl algebra.

(c) The function fields of the affine quantum planes (see Example 10.1.1).

(d) The function fields of the three dimensional Sklyanin algebras.

Since both (b) and (c) are special cases of division rings of skew polynomial rings, this confirms the conjecture of Artin from 10.1 in this case.

13.4. Noncommutative quadrics. Now let $X$ be a noncommutative quadric as defined in $\$ 11.3$. There exist naturally defined objects $\mathcal{O}_{X}(n, n)$ (analogues of $\left.\mathcal{O}_{\mathbb{P}^{1}}(n) \otimes \mathcal{O}_{\mathbb{P}^{1}}(n)\right)$ and we put $\mathcal{L}_{n}=\mathcal{O}_{X}(n, n)$.

One can show that the elliptic curve $Y$ used in the construction of $X$ actually embeds as a divisor in $X$. Let $d=p$ be a point in $Y$. Then $\widetilde{X}$ turns out to be a noncommutative $\mathbb{P}^{2}$. From this one deduces the following result. 
Theorem 13.4.1. Every noncommutative quadric is birational to a noncommutative $\mathbb{P}^{2}$.

One can prove the converse of this theorem using similar methods.

13.5. Noncommutative cubic surfaces. We again let $X$ be a nontrivial noncommutative $\mathbb{P}^{2}$, but now we put $\mathcal{L}_{n}=\mathcal{O}_{X}(3 n)$ and we take six points $\left\{p_{1}, \ldots, p_{6}\right\} \in Y$ in sufficiently general position. Put $d=\sum_{i} p_{i}$. Then it follows from 87 that there exists a graded ring $S$, with the Hilbert series of a polynomial ring in 4 variables and containing a normal non-zero divisor $g$ of degree three such that $\widetilde{X}=\mathrm{QGr}(S /(g))$. In other words, $\widetilde{X}$ can be considered as a noncommutative cubic surface in a noncommutative $\mathbb{P}^{3}$.

In each of these examples, (13.3)-(13.5), the analogy with the commutative case is striking.

13.6. More details on Example 10.1.1. We end the paper by showing how to use the techniques of this section to prove the result asserted in that example. Recall that $E=k(u, v ; v u=q u v)$ and $D=E^{\tau}$ where $\tau u=u^{-1}, \tau v=v^{-1}$. The problem is to determine where $D$ fits in Artin's list of division rings from $\$ 10.1$

Put $L=k(u)$ and let $K$ be the subfield of $L$ given by $k(s)=k\left(u+u^{-1}\right)$. Define $\psi \in \operatorname{Aut}(L)$ by $\psi(u)=\theta u$ where $\theta$ is a scalar satisfying $\theta^{-2}=q$. Let $M$ be the $K-K$ bimodule given by id $L_{\psi}$. Thus $s$ acts on the right of $M$ by $t=\theta u+\theta^{-1} u^{-1}$. An explicit computation now shows that $D$ is the function field of the $\mathbb{P}^{1}$-bundle over Spec $K$ associated to $M$.

We may identify $M$ with the $k(s) \otimes_{k} k(t)$-module $k(u)$ where $s$ and $t$ act in the way indicated above. We will now view $K$ as the function field of $\mathbb{P}^{1}$ and we will identify $M$ with the localization at the generic point of a sheaf-bimodule $\mathcal{M}$. To do this note that the images of $s$ and $t$ in $k(u)$ satisfy the equation

$$
(\theta s-t)\left(\theta^{-1} s-t\right)=-\left(\theta-\theta^{-1}\right)^{2} .
$$

Let $Y \subset \mathbb{P}^{1} \times \mathbb{P}^{1}$ be the closure of the curve in Spec $k[s, t]$ defined by (13.1). Closer inspection reveals that $Y$ is in fact a nodal elliptic curve with a unique singular point at $(\infty, \infty)$.

We now define $\mathcal{M}={ }_{\mathrm{pr}_{1}} \mathcal{L}_{\mathrm{pr}_{2}}$ where $\mathcal{L}$ is a generic line bundle of degree two on $Y$. Thus $D$ is the function field of the $\mathbb{P}^{1}$-bundle over $\mathbb{P}^{1}$ associated to $\mathcal{M}$. By Theorem 12.6 this $\mathbb{P}^{1}$-bundle is isomorphic to a noncommutative quadric and, by 13.4 above, a noncommutative quadric is birational to a noncommutative plane $X$. If we examine more closely the birational equivalence in 13.4 then this noncommutative plane happens to be associated to a triple $(Y, \mathcal{L}, \sigma)$ where $Y$ is a triangle. Thus the function field of $X$ is the same as that of an affine quantum plane and we are done.

Of course, once one knows that $D$ is the function field of a quantum plane, it should not be too difficult to write down the requisite generators and recently we have succeeded in achieving this. Set

$$
f=\left(u v-u^{-1} v^{-1}\right)\left(v-v^{-1}\right)^{-1} \quad \text { and } \quad g=\left(u-u^{-1}\right)\left(v-v^{-1}\right)^{-1} .
$$

Then one can show that $f g=q g f$ and that $D$ is the division ring of fractions of the quantum plane $k\langle f, g\rangle$. In particular, $D \cong E$. Proving this is a long but elementary computation. 


\section{INDEX}

Much of the basic notation can be found in the Notation Section 1.1 and concepts defined there are not repeated in this index.

ample divisor, 177

ample pair $(\mathcal{O}, s), 178$

AS Gorenstein, 194

AS regular, 195

$\operatorname{bimod}\left(\mathcal{O}_{X}-\mathcal{O}_{Y}\right), 207$

birational equivalence, 174

$\chi$ condition, 179

classical Serre duality, 193

cohomological dimension, 192

exceptional curve, 198

exceptional object, 203

Ext-finite, 190

finite length object, 193

Gelfand-Kirillov dimension, 182

Goldie ring, 188

hereditary category, 190

homological dimension $n, 190$

maximal order, 194 nice ring, 188

noncommutative $\mathbb{P}^{1}$-bundle, 208

noncommutative quadric, 205

(nontrivial) noncommutative $\mathbb{P}^{2}, 203$

one-critical object, 192

$\mathbb{P}^{1}$-bundle $\mathbb{P}(\mathcal{E}), 206$

$\pi(M), 179$

point scheme $\mathcal{P}(A), 196$

quasi-unipotent automorphism, 181

$R_{\lambda}, 198$

saturated, 192

Serre duality, Serre functor, 190

$\sigma$-ample divisor, 180

Sklyanin algebras $\mathrm{Skl}_{3}, \mathrm{Skl}_{4}, 197$, twisted coordinate ring, 180

twisting ring, 187

Veronese ring, 184

$\mathbb{Z}$-algebra, 202

\section{REFERENCES}

[1] K. Ajitabh, Residue complex for Sklyanin algebras of dimension three, Adv. Math., 144 (1999), 137-160. CMP 99:14

[2] M. Artin, Some problems on three-dimensional graded domains, Representation theory and algebraic geometry, London Math. Soc. Lecture Note Ser., vol. 238, Cambridge Univ. Press, 1995, pp. 1-19. MR 99a:16037

[3] M. Artin and W. Schelter, Graded algebras of global dimension 3, Adv. in Math. 66 (1987), 171-216. MR 88k:16003

[4] M. Artin and J. T. Stafford, Noncommutative graded domains with quadratic growth, Invent. Math. 122 (1995), 231-276. MR 96g:16027

[5] _ Semiprime graded algebras of dimension two, J. Algebra, 277 (2000). 68-123. CMP 2000:12

[6] M. Artin, J. Tate, and M. Van den Bergh, Some algebras associated to automorphisms of elliptic curves, The Grothendieck Festschrift, vol. 1, Birkhäuser, Boston, 1990, pp. 33-85. MR 92e: 14002

[7] — Modules over regular algebras of dimension 3, Invent. Math. 106 (1991), 335-388. MR 93e:16055

[8] M. Artin and M. Van den Bergh, Twisted homogeneous coordinate rings, J. Algebra 133 (1990), 249-271. MR 91k:14003

[9] M. Artin and J. J. Zhang, Noncommutative projective schemes, Adv. in Math. 109 (1994), no. 2, 228-287. MR 96a:14004

[10] _ Abstract Hilbert schemes, Algebr. Represent. Theory, to appear.

[11] M. F. Atiyah and I. G. Macdonald, Introduction to Commutative Algebra, Addison-Wesley, London, 1969. MR 39:4129

[12] S. Barannikov and M. Kontsevich, Frobenius manifolds and formality of Lie algebras of polyvector fields., Internat. Math. Res. Notices 4 (1998), 201-215. MR 99b:14009

[13] W. Barth, C. Peters, and A. Van de Ven, Compact Complex Surfaces, Ergebnisse der Mathematik und Ihrer Grenzgebiete, vol. 3, Springer Verlag, Berlin, 1984. MR 86c:32026

[14] A. Beauville, Surface algébriques complexes, Astérisque, 54 (1978). [MR 58:5686

[15] A. Beilinson and J. Bernstein, Localisation de g-modules, C. R. Acad. Sci. Paris Sér. I Math. 292 (1981), 15-18. MR 82k:14015 
[16] A. I. Bondal, Noncommutative deformations and Poisson brackets on projective spaces, MPIpreprint, 1993.

[17] A. I. Bondal and M. M. Kapranov, Representable functors, Serre functors, and reconstructions, Izv. Akad. Nauk SSSR Ser. Mat. 53 (1989), no. 6, 1183-1205, 1337. MR 91b:14013

[18] A. I. Bondal and D. O. Orlov, Semi-orthogonal decompositions for algebraic varieties, MPI preprint, 1996.

[19] A. I. Bondal and A. E. Polishchuk, Homological properties of associative algebras: the method of helices, Russian Acad. Sci. Izv. Math. 42 (1994), 219-260. MR 94m:16011

[20] A. I. Bondal and M. Van den Bergh, in preparation.

[21] P. M. Cohn, Algebra, John Wiley \& Sons, New York, 1982. MR 83e:00002

[22] P. Gabriel, Des catégories abéliennes, Bull. Soc. Math. France 90 (1962), 323-448. MR 38:1144

[23] I. M. Gel'fand and A. A. Kirillov, Sur les corps liés aux algèbres enveloppantes des algèbres de Lie, Inst. Hautes Études Sci. Publ. Math. 31 (1966), 5-19. MR 34:7731

[24] K. R. Goodearl and R. B. Warfield, Jr., An Introduction to Noncommutative Noetherian Rings, London Math. Soc. Student Texts, vol. 16, Cambridge University Press, 1989. MR 91c:16001

[25] A Grothendieck, Sur quelques points d'algèbre homologique, Tôhoku Math. J., 9 (1957), 119-221. MR 21:1328

[26] R. Hartshorne, Algebraic Geometry, Springer Verlag, Berlin, 1977. MR 57:3116

[27] T. J. Hodges, Morita equivalence of primitive factors of u(sl(2)), Contemp. Math. 139 (1992), 175-179. MR 94e:17007

[28] P. Jorgensen, Serre duality for Tails $(A)$, Proc. Amer. Math. Soc. 125 (1997), 709-716. MR 97e:14002

[29] _ Noncommutative graded homological identities, J. London Math. Soc. 57 (1998), 336-350. MR 99h:16010

[30] _ Intersection theory on noncommutative surfaces, Trans. Amer. Math. Soc., 352 (2000), 5817-5854. CMP 99:14

[31] P. Jorgensen and J. J. Zhang, Gourmet Guide to Gorensteinness, Adv. Math., 151 (2000), 313-345. CMP 2000:12

[32] M. Kapranov, Non-commutative geometry based on commutator expansions, J. Reine Angew. Math. 505 (1998), 73-118. MR 2000b:14003

[33] A. Kapustin, A. Kuznetsov, D. Orlov, Noncommutative Instantons and Twistor Transform, hep-th/0002193, 2000.

[34] D. S. Keeler, Criteria for $\sigma$-ampleness, J. Amer. Math. Soc., 13 (2000), 517-532. CMP 2000: 12

[35] B. Keller, Deriving DG-categories, Ann. Sci. École Norm. Sup. (4) 27 (1994), 63-102. MR 95h:18014

[36] Introduction to $A_{\infty}$ algebras and modules, to appear; math.RA/9910179, 1999.

[37] G. M. Kelly and R. Street, Review of the elements of 2-categories, Category Seminar, Lecture Notes in Math., vol. 420, Springer Verlag, Berlin, 1972, pp. 75-103. MR 50:10010

[38] S. L. Kleiman, Toward a numerical theory of ampleness, Ann. Math. 84 (1966), 293-344. MR 34:5834

[39] M. Kontsevich and A. Rosenberg, Noncommutative smooth spaces, The Gelfand Mathematical Seminars, 1996-1999, 85-108, Gelfand Math. Sem., Birkhäuser Boston, Boston, MA, 2000. CMP 2000:17

[40] G. R. Krause and T. H. Lenagan, Growth of Algebras and Gelfand-Kirillov Dimension, Research Notes in Math., vol. 116, Pitman, Boston, 1985. MR 86g:16001

[41] L. Le Bruyn, Noncommutative geometry at n, math.AG/9904171, 1999.

[42] _ Non-commutative compact manifolds constructed from quivers., AMA Algebra Mpntp. Announc., 1999, No.1. CMP 2000:07

[43] L. Le Bruyn, S. P. Smith, and M. Van den Bergh, Central extensions of three-dimensional Artin-Schelter regular algebras, Math. Z. 222 (1996), 171-212. MR 98c:16028

[44] T. Levasseur and S. P. Smith, Modules over the 4-dimensional Sklyanin algebra, Bull. Soc. Math. France 121 (1993), 35-90. MR 94f:16054

[45] Y. I. Manin, Cubic forms: Algebra, Geometry, Arithmetic, North Holland, Amsterdam, 1974. MR 57:343 
[46] —, Quantum groups and noncommutative geometry, Montreal University, 1988. MR 91e:17001

[47] - Topics in Noncommutative Geometry, Princeton University Press, New Jersey, 1991. MR 92k:58024

[48] J. C. McConnell and J. C. Robson, Noncommutative Noetherian Rings, John Wiley \& Sons, New York, 1987. MR 89j:16023

[49] B. Mitchell, Rings with several objects, Advances in Math. 8 (1972), 1-161. MR 45:3524

[50] C. Năstăsescu and F. Van Oystaeyen, Graded Ring Theory, North Holland, Amsterdam, 1982. MR 84i: 16002

[51] A. Neeman, The Grothendieck duality theorem via Bousfield's techniques and Brown representability, J. Amer. Math. Soc. 9 (1996), 205-236. MR 96c:18006

[52] A. V. Odesskii and B. L. Feigin, Sklyanin algebras associated with an elliptic curve, preprint, Institute for Theoretical Physics, Kiev, 1989.

[53] _ Sklyanin's elliptic algebras, Functional Anal. Appl. 23 (1989), no. 3, 207-214. MR 91e:16037

[54] D. M. Patrick, Noncommmutative Ruled Surfaces, Ph.D. Thesis, MIT, June 1997;

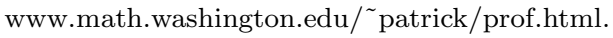

[55] _ Noncommutative symmetric algebras of two-sided vector spaces, J. Algebra 223 (2000), 16-36.

[56] I. Reiner, Maximal Orders, Academic Press, New York, 1975. MR 52:13910

[57] I. Reiten and M. Van den Bergh, Noetherian hereditary categories satisfying Serre duality, to appear; math.RT/9911242, 1999.

[58] R. Resco, A dimension theorem for division rings, Israel J. Math. 35 (1980), no. 3, 215-221. MR 81j:16021

[59] C. M. Ringel, Tame algebras and integral quadratic forms, Lecture Notes in Mathematics, vol. 1099, Springer Verlag, Berlin, 1984. MR 87f:16027

[60] A. L. Rosenberg, Noncommutative Algebraic Geometry and Representations of Quantized Algebras, Mathematics and its Applications, vol. 330, Kluwer Academic Publishers, Dordrecht, 1995. MR 97b:14004

[61] _ The spectrum of abelian categories and reconstruction of schemes, Rings, Hopf algebras, and Brauer groups, Lecture Notes in Pure and Appl. Math., vol. 197, Marcel Dekker, New York, 1998, pp. 257-274. MR 99d:18011

[62] L. H. Rowen, Polynomial Identities in Ring Theory, Academic Press, New York, 1980. MR 82a: 16021

[63] A. H. Schofield, Stratiform simple Artinian rings, Proc. London Math. Soc. (3) 53 (1986), no. 2, 267-287. MR 87i:16026

[64] J. P. Serre, Faisceaux algébriques cohérents, Ann. of Math. (2) 61 (1955), 197-278. MR 16:953c

[65] I. R. Shafarevitch, Basic Algebraic Geometry I, Springer Verlag, Berlin, 1994.

[66] B. Shelton and M. Vancliff, Embedding a quantum rank three quadric in a quantum $\mathbb{P}^{3}$, Comm. Algebra 27 (1999), 2877-2904. CMP 99:12

[67] E. K. Sklyanin, Some algebraic structures connected to the Yang-Baxter equation, Functional Anal. Appl. 16 (1982), 27-34. MR 84c:82004

[68] _ Some algebraic structures connected to the Yang-Baxter equation. Representations of quantum algebras, Functional Anal. Appl. 17 (1983), 273-284. MR 85k:82011

[69] L. W. Small, J. T. Stafford, and R. B. Warfield, Affine algebras of Gelfand-Kirillov dimension one are PI, Math. Proc. Camb. Phil. Soc. 97 (1985), 407-414. MR 86g:16025

[70] L. W. Small and R. B. Warfield, Jr, Prime affine algebras of Gelfand-Kirillov dimension one, J. Algebra 91 (1984), 386-389. MR 86h:16006

[71] S. P. Smith, The 4-dimensional Sklyanin algebras, K-theory 8 (1994), 65-80. MR 95i:16016

[72] S. P. Smith and J. T. Stafford, Regularity of the 4-dimensional Sklyanin algebra, Compositio Math. 83 (1992), 259-289. MR 93h:16037

[73] S. P. Smith and J. M. Staniszkis, Irreducible representations of the 4-dimensional Sklyanin algebra at points of infinite order, J. Algebra 160 (1993), 57-86. MR 95c:16027

[74] S. P. Smith and J. J. Zhang, Curves on noncommutative schemes, Algebr. Represent. Theory 1 (1998), 311-351. CMP 99:11

[75] J. T. Stafford, Regularity of algebras related to the Sklyanin algebras, Trans. Amer. Math. Soc. 341 (1994), no. 2, 895-916. MR 94d:16040 
[76] J. T. Stafford and J. J. Zhang, Examples in noncommutative projective geometry, Math. Proc. Cambridge Philos. Soc. 116 (1994), 415-433. MR 95h:14001

[77] _ Homological properties of (graded) noetherian PI rings, J. Algebra, 168 (1994), 988-1026. MR 95h:16030

[78] B. Stenström, Rings of Quotients, Die Grundlehren der mathematischen Wissenschaften in Einzeldarstellungen, vol. 217, Springer Verlag, Berlin, 1975. MR 52:10782

[79] D. R. Stephenson, Artin-Schelter regular algebras of global dimension three, J. Algebra 183 (1996), 55-73. MR 97h:16053

[80] , Algebras associated to elliptic curves, Trans. Amer. Math. Soc. 349 (1997), 23172340. MR 97m:16080

[81] D. R. Stephenson and J. J. Zhang, Noetherian connected graded algebras of global dimension 3, J. Algebra, 230 (2000), 474-495. CMP 2000:16

[82] J. Tate and M. Van den Bergh, Homological properties of Sklyanin algebras, Invent. Math. 124 (1996), 619-647. MR 98c:16057

[83] R. Thomason and T. Trobaugh, Higher algebraic $K$-theory of schemes and of derived categories, The Grothendieck Festschrift, vol. 3, Birkhäuser, Boston, 1990, pp. 247-435. MR 92f:19001

[84] M. Vancliff and K. Van Rompay, Embedding a quantum non-singular quadric in a quantum $\mathbb{P}^{3}$, J. Algebra 195 (1997), 93-129. MR 99a:16040

[85] M. Vancliff, K. Van Rompay and L. Willaert, Some quantum $\mathbb{P}^{3}$ s with finitely many points, Comm. Algebra 26 (1998), 1193-1208. MR 99c:16045

[86] M. Van den Bergh, A translation principle for Sklyanin algebras, J. Algebra 184 (1996), 435-490. MR 98a:16047

[87] _ Blowing-up of noncommutative smooth surfaces, Mem. Amer. Math. Soc., to appear; math.QA/980911, 1998.

[88] Noncommutative quadrics, in preparation.

[89] Some noncommutative birational transformations, in preparation.

[90] M. Van den Bergh and M. Van Gastel, Graded modules of Gelfand-Kirillov dimension one over three-dimensional Artin-Schelter regular algebras, J. Algebra 196 (1997), 251-282. MR 99c:16020

[91] F. Van Oystaeyen and A. Verschoren, Noncommutative Algebraic Geometry, Lecture Notes in Math., vol. 887, Springer Verlag, Berlin, 1981. MR 85i:16006

[92] F. Van Oystaeyen and L. Willaert, Examples and quantum sections of schematic algebras, J. Pure Appl. Algebra 120 (1997), no. 2, 195-211. MR 98h:14004

[93] A. B. Verevkin, On a non-commutative analogue of the category of coherent sheaves on a projective scheme, Amer. Math. Soc. Transl. 151 (1992), 41-53. MR 93j:14002

[94] Q. S. Wu and J. J. Zhang, Some homological invariants of local PI algebras, J. Algebra 225 (2000), 904-935. CMP 2000:09

[95] A. Yekutieli and J. J. Zhang, Serre duality for noncommutative projective schemes, Proc. Amer. Math. Soc. 125 (1997), 697-707. MR 97e:14003

[96] _ Rings with Auslander dualizing complexes, J. Algebra 213 (1999), 1-51. MR 2000f:16012

[97] J. J. Zhang, On lower transcendence degree., Adv. Math. 139 (1998), 157-193. MR 2000a:16046

Department of Mathematics, University of Michigan, Ann Arbor, Mi 48109

E-mail address: jts@math.lsa.umich.edu

Departement WNi, Limburgs Universitair Centrum, 3590 Diepenbeek, Belgium

E-mail address: vdbergh@luc.ac.be 